OPEN ACCESS

Edited by:

Andrea Nistri,

Scuola Internazionale di Studi

Superiori Avanzati (SISSA), Italy

Reviewed by: Nicola Berretta, Fondazione Santa Lucia (IRCCS),

Rory McQuiston, Virginia Commonwealth University, United States

${ }^{*}$ Correspondence: Annamaria Morelli a.morelli@unifi.it

Received: 18 July 2017 Accepted: 13 October 2017 Published: 27 October 2017

Citation:

Morelli A, Sarchielli E, Guarnieri G, Coppi E, Pantano D, Comeglio P, Nardiello P, Pugliese AM, Ballerini L, Matucci R, Ambrosini $S$, Castronovo G, Valente R, Mazzanti B, Bucciantini S, Maggi M, Casamenti F, Gallina $P$ and Vannelli GB (2017) Young Human Cholinergic Neurons Respond to Physiological Regulators and Improve Cognitive Symptoms in an Animal Model of Alzheimer's Disease.

Front. Cell. Neurosci. 11:339. doi: 10.3389/fncel.2017.00339

\section{Young Human Cholinergic Neurons Respond to Physiological Regulators and Improve Cognitive Symptoms in an Animal Model of Alzheimer's Disease}

\author{
Annamaria Morelli ${ }^{1 *}$, Erica Sarchielli ${ }^{1}$, Giulia Guarnieri ${ }^{1}$, Elisabetta Coppi ${ }^{2}$, \\ Daniela Pantano ${ }^{2}$, Paolo Comeglio ${ }^{3}$, Pamela Nardiello ${ }^{2}$, Anna M. Pugliese ${ }^{2}$, \\ Lara Ballerini ${ }^{4}$, Rosanna Matucci ${ }^{2}$, Stefano Ambrosini ${ }^{1}$, Giuseppe Castronovo ${ }^{5}$, Rosa \\ Valente ${ }^{2}$, Benedetta Mazzanti ${ }^{4}$, Sandra Bucciantini ${ }^{6}$, Mario Maggi ${ }^{3}$, Fiorella Casamenti ${ }^{2}$, \\ Pasquale Gallina ${ }^{7}$ and Gabriella B. Vannelli ${ }^{1}$
}

\begin{abstract}
'Section of Human Anatomy and Histology, Department of Experimental and Clinical Medicine, University of Florence, Florence, Italy, ${ }^{2}$ Department of Neuroscience, Psychology, Drug Research and Child Health, Division of Pharmacology and Toxicology, University of Florence, Florence, Italy, ${ }^{3}$ Sexual Medicine and Andrology Unit, Department of Experimental and Clinical Biomedical Sciences "Mario Serio", University of Florence, Florence, Italy, ${ }^{4}$ Cell Therapy and Transfusion Medicine Unit, Department of Experimental and Clinical Medicine, University of Florence, Florence, Italy, ${ }^{5}$ Department of Experimental and Clinical Biomedical Sciences "Mario Serio", Section of Clinical Physiopathology, Florence, Italy, ${ }^{6}$ Careggi University Hospital, Florence, Italy, ${ }^{7}$ Neurosurgery School of Tuscany, Department of Surgery and Translational Medicine, University of Florence, Florence, Italy
\end{abstract}

The degeneration of cholinergic neurons of the nucleus basalis of Meynert (NBM) in the basal forebrain (BF) is associated to the cognitive decline of Alzheimer's disease (AD) patients. To date no resolutive therapies exist. Cell-based replacement therapy is a strategy currently under consideration, although the mechanisms underlying the generation of stem cell-derived NBM cholinergic neurons able of functional integration remain to be clarified. Since fetal brain is an optimal source of neuronal cells committed towards a specific phenotype, this study is aimed at isolating cholinergic neurons from the human fetal NBM (hfNBMs) in order to study their phenotypic, maturational and functional properties. Extensive characterization confirmed the cholinergic identity of hfNBMs, including positivity for specific markers (such as choline acetyltransferase) and acetylcholine (Ach) release. Electrophysiological measurements provided the functional validation of hfNBM cells, which exhibited the activation of peculiar sodium $\left(\mathrm{I}_{\mathrm{Na}}\right)$ and potassium $\left(\mathrm{I}_{\mathrm{K}}\right)$ currents, as well as the presence of functional cholinergic receptors. Accordingly, hfNBMs express both nicotinic and muscarinic receptors, which were activated by Ach. The hfNBMs cholinergic phenotype was regulated by the nerve growth factor (NGF), through the activation of the high-affinity NGF receptor TrkA, as well as by $17-\beta$-estradiol through a peculiar recruitment of its own receptors. When intravenously administered in NBM-lesioned rats, hfNBMs determined a significant improvement in memory functions. Histological examination of brain sections showed that hfNBMs (labeled with $\mathrm{PKH} 26$ fluorescent dye prior to administration) reached the damaged brain areas. 
The study provides a useful model to study the ontogenetic mechanisms regulating the development and maintenance of the human brain cholinergic system and to assess new lines of research, including disease modeling, drug discovery and cell-based therapy for AD.

Keywords: nucleus basalis of Meynert, cell-based therapy, NGF, estrogen receptors, primary cilium, cholinergic receptors

\section{INTRODUCTION}

The basal forebrain (BF) region is a broad topographic term describing a heterogeneous set of cellular structures on the medial and ventral cerebral hemisphere. It comprises the nucleus basalis of Meynert (NBM), the vertical and horizontal diagonal bands of Broca and the medial septal nucleus (Mesulam, 2013). This complex region contains magnocellular neurons that provide the major cholinergic projections to the cerebral cortex, hippocampus and amygdala. The BF cholinergic system has been implicated in the modulation of an ever-expanding set of behavioral states, including attention, memory and learning (Ferreira-Vieira et al., 2016). Importantly, the degeneration and loss of cholinergic neurons within the $\mathrm{BF}$, especially in the $\mathrm{NBM}$, represents a pathological correlate of the well-documented cholinergic derangement in Alzheimer's disease (AD) patients (Kilimann et al., 2014).

Despite its implications as pathological substrate for cholinergic deficiency in $\mathrm{AD}$ brain, very little is known about the developmental properties of the NBM neuronal contingent during embryogenesis. As we move towards an era of stem cell-based treatments for several pathologies, including neurodegenerative disorders, the use of cholinergic cell-based transplantation approaches to treat $\mathrm{AD}$ should be considered. However, extensive studies are needed to identify the mechanisms underlying the generation of functionally integrated stem cell-derived NBM cholinergic neurons. Since fetal brain is an optimal resource to study developmental processes of neurons already committed towards a specific phenotype, the aim of the present study is the isolation and characterization of human cholinergic neurons from the fetal NBM.

Studies in animal models after fetal tissue transplantation have demonstrated that neuronal replacement and partial reconstruction of damaged neuronal circuitry is possible (Björklund and Lindvall, 2000). There is also evidence from clinical trials that fetal graft in the diseased human brain can lead to symptomatic relief (Paganini et al., 2014). Human neurological disorders such as AD, Parkinson's disease (PD), Huntington's disease, stroke or spinal cord injury are caused by the loss of neurons and glial cells in the brain or spinal cord. In each of them a different spectrum of cell types is affected; therefore, before clinical trials are initiated, we need to know much more about how to control cell proliferation and differentiation into specific phenotypes, promote their integration into existing neural and synaptic circuits, and determine functional recovery in animal models closely resembling the human disease. Recently,
Grealish et al. (2014) have provided preclinical evidence that the potency of the human embryonic stem cell-derived dopaminergic neurons, with respect to functional efficacy and long-distance targeted reinnervation, is comparable to that of fetal ventral mesencephalon cells, which provides important support for their therapeutic potential and use for cell replacement therapy in $\mathrm{PD}$ patients.

The NBM precursor neurons were reported to originate in the telencephalic subpallium (Xu et al., 2008). A new pallial origin of these neurons has been also described (Pombero et al., 2011). These differences in the BF cholinergic system ontogenesis might explain their different connection patterns (cortex vs. hippocampus) and functions in cognitive processes, as well as their specific responses to pathological stimuli. The data on the development and anatomical organization of the NBM in the human fetal brain revealed early maturation. The earliest signs of the acetylcholinesterase (AchE) activity, indicative of acetylcholine (Ach) release, appear as early as 9 weeks of gestation (w.g.) in the area where the prospective basal nucleus develops and the first AChE-reactive bundles form between this age and 10.5 w.g. (Kostović, 1986). At 12-15 weeks, the positive cell group appear and the basal nucleus can be separated into several subdivisions. Innervation of the developing cortex and subcortical areas by AchE-reactive fibers occurs between 16 w.g. and 28 w.g. An overall high degree of structural and chemical complexity is early achieved in the NBM of human fetal brain (Kračun and Rösner, 1986), suggesting that this brain area plays an important role in the earliest cortical connections due to its specific functional entity. Of great importance for the definition of the NBM within the human brain was the demonstration that this nucleus increases both in its relative and absolute size and differentiation with increasing cerebralization (Gorry, 1963).

In this work, we isolated neurons from NBM of 12 week old human fetuses, which is the proper time during embryogenesis when the commitment into differentiation programs for $\mathrm{BF}$ nuclear complex is well defined (Kostović, 1986). Although retaining growth and differentiation potentials, an extensive phenotypical and functional characterization demonstrated that, in the chosen "developmental window", these neurons possess a clear BF cholinergic identity, which may be importantly induced and maintained by physiological factors, such as nerve growth factor (NGF) and estrogens. More importantly, when intravenously injected into NBM-lesioned rats these cells reached the damaged brain area and ameliorated cognitive deficiencies typical of $\mathrm{AD}$. These results provide the first characterization of human fetal BF cholinergic neurons and show their potential as a useful resource in the research field of cognitive disorders. 


\section{MATERIALS AND METHODS}

Further information can be found in Supplementary Material.

\section{Cell Culture}

The use of human fetal tissue for research purposes was approved by the National Ethics Committee and the local ethic committee for investigation in Humans of the University of Florence (Permit Number: 678304). Human fetuses biopsies were obtained from therapeutic medical abortions after women approved and signed the informed consent document, as already reported (Gallina et al., 2008). NBM tissue was dissected from two female 12-weeks old human fetuses and incubated with $1 \mathrm{mg} / \mathrm{ml}$ collagenase type IV (Sigma-Aldrich Corp., St. Louis, MO, USA). The cell suspensions were mechanically dispersed by pipetting and cultured in Coon's modified Ham's F12 medium (Euroclone, Milan, Italy) supplemented with 10\% FBS (Hyclone, Logan, UT, USA). Cells were used within the 26th passage.

\section{Quantitative Real-Time RT-PCR}

Isolation of total RNA and cDNA synthesis were performed using the "RNeasy Micro kit" (Qiagen, Hilden, Germany) and the iScript ${ }^{\mathrm{TM}}$ cDNA Synthesis Kit (Bio-Rad Laboratories, Hercules, CA, USA). For some genes, quantitative real time PCR (qRTPCR) was performed according to the fluorescent TaqMan methodology, as previously described (Morelli et al., 2008) Primers and probes for the target genes were predeveloped assays (Life Technologies, Carlsbad, CA, USA) as listed in Supplementary Table S1. For the remaining genes, qRT-PCR was performed using SsoFast ${ }^{\mathrm{TM}}$ EvaGreen ${ }^{\circledR}$ Supermix (BioRad Laboratories) as previously described (Morelli et al., 2013). Specific primers sequences for the target genes are reported in Supplementary Table S2. The 18S ribosomal RNA subunit was chosen as the housekeeping gene for relative quantification. Data analysis was based on the comparative threshold cycle $(\mathrm{Ct})$ using the $2^{-\Delta \Delta \mathrm{Ct}}$ method and carried out with the MyIQ2 ${ }^{\mathrm{TM}}$ Two-Color Real-Time PCR Detection System (Bio-Rad Laboratories).

\section{Immunofluorescence}

The analysis was performed as previously described (Sarchielli et al., 2014) using the following primary antibodies: anti-ChAT polyclonal antibody (pAb; 1:200; Millipore, Temecula, CA, USA), anti-VAchT pAb (1:1000), anti-acetylated $\alpha$-tubulin $\mathrm{mAb}$ (1:500) from Sigma-Aldrich Corp., St. Louis, MO, USA; anti-GFAP monoclonal antibody (mAb; 1:100), anti- $\alpha$ tubulin $\mathrm{mAb}$ (1:2000), anti-ER $\alpha \mathrm{mAb}$ (1:50), anti-ER $\beta \mathrm{mAb}$ (1:50) from Santa Cruz Biotechnology (Santa Cruz, CA, USA); anti-GPR30 pAb (1:40, Abcam, Cambridge, UK), followed by Alexa Fluor 488 or 568 goat anti-rabbit or Alexa Fluor 488 goat anti-mouse (1:200, Molecular Probes, Eugene, Oregon), as appropriate. For $\alpha$-tubulin and acetylated $\alpha$-tubulin staining, cells were cultured in serum/phenol red-free condition and treated with NGF $(100 \mathrm{ng} / \mathrm{ml}), \mathrm{E} 2(10 \mathrm{nM})$ or $\mathrm{G} 1(100 \mathrm{nM})$ for $24 / 48 \mathrm{~h}$ in presence or absence of the receptor inhibitors K252a (200 nM), Tamoxifen $(100 \mathrm{nM})$ or G15 $(1 \mu \mathrm{M})$, respectively. The number of cells with neurites longer than four times the cell body
( $\alpha$-tubulin staining) or the number of ciliated cells (acetylated $\alpha$-tubulin staining) was calculated by counting the stained cells in ten fields per slide of three different experiments performed in triplicate.

\section{Flow Cytometry}

As previously described (Sarchielli et al., 2017), after fixation and permeabilization, cells were resuspended in PBS with $1 \%$ FBS and incubated with the following primary antibodies: anti-O4 mAb (1:100), anti-MAP2 pAb (1:100), anti-ChAT pAb (1:100) from Millipore; anti-GFAP mAb (1:100), anti-TrkA pAb (1:100) from Santa Cruz Biotechnology, followed by incubation with Alexa Fluor 488 goat anti-mouse IgM (1:200), Alexa Fluor 568 goat anti-rabbit or Alexa Fluor 488 goat anti-mouse IgG $(\mathrm{H}+\mathrm{L} ; 1: 200)$ secondary antibodies from Molecular Probes, as appropriate. Cells were analyzed on a FACSCanto II instrument (BD Pharmingen, San Diego, CA, USA) using BD FACSDiva (BD) and FlowJo v10 (Tree Star, Inc., Ashland, OR, USA) softwares.

\section{Acetylcholine Release Assay}

Cells were cultured in choline chloride free medium (SigmaAldrich Corp., St. Louis, MO, USA) until subconfluence, then the Ach release in the culture medium was quantified by Choline/Acetylcholine Assay Kit (Abcam), according to the manufacturer's instructions and using a FlexStation 3 Microplate Reader (Molecular Devices, Sunnyvale, CA, USA).

\section{Western Blot Analysis}

Cells were cultured in serum/phenol red-free condition and treated with NGF $(10-100 \mathrm{ng} / \mathrm{ml}), \mathrm{E} 2\left(10^{-10}-10^{-7} \mathrm{M}\right)$ or G1 $(100 \mathrm{nM})$ in presence or absence of the receptor inhibitors K252a (200 nM), Tamoxifen (100 nM) or G15 (1 $\mu \mathrm{M})$. Protein extracts $(20 \mu \mathrm{g})$ were subjected to immunoblotting as previously described (Sarchielli et al., 2014) using the following primary antibodies: anti-p-TrkA pAb (1:1000), anti-pERK $1 / 2 \mathrm{mAb}(1: 1000)$ from Cell Signaling Technologies (Danvers, MA, USA); anti-p-CREB pAb (1:1000), anti- $\alpha$-tubulin mAb (1:2000), anti- $\beta$ actin $\mathrm{mAb}$ (1:10000), anti-GAP43 $\mathrm{mAb}$ (1:1000), anti-STAT1 pAb (1:1000) from Santa Cruz Biotechnology; anti-c fos pAb (1:1000, Sigma-Aldrich); anti-ChAT pAb (1:2000, Millipore).

\section{Electrophysiology}

Whole-cell patch-clamp recordings were performed in $-60 \mathrm{mV}$ clamped-cells by using the following solutions: extracellular (mM): HEPES 10, D-glucose 5, $\mathrm{NaCl} 140, \mathrm{KCl} 3, \mathrm{MgCl}_{2}$ 2, and $\mathrm{CaCl}_{2} 2(\mathrm{pH}$ 7.4). Pipette (mM): K-Aspartate 130, $\mathrm{MgCl}_{2} 2, \mathrm{Na}_{2}$-ATP 5, $\mathrm{Na}_{2}$-GTP 0.1, EGTA 11, HEPES 10 (pH 7.2). Data were acquired with an Axopatch 200B amplifier (Axon Instruments, Union City, CA, USA), low-pass filtered at $10 \mathrm{kHz}$, stored and analyzed with a pClamp 9.2 software (Axon Instruments). Detailed protocols used to evoke voltagedependent $\mathrm{K}^{+}$or $\mathrm{Na}^{+}$currents are described in Supplementary Material. Drugs used were applied by superfusion with a three-way perfusion valve controller (Harvard Apparatus). Current-clamp recordings were performed by applying 12 steps 
of current injection (300 ms duration; $100 \mathrm{pA}$ increment, from $-100 \mathrm{pA}$ to $1000 \mathrm{pA}$ ) from the resting membrane potential of the investigated cell, as already described (Coppi et al., 2012) and detailed in Supplementary Material.

\section{Radioligand Binding Assays}

Cell membrane preparations $(0.5-0.7 \mathrm{mg} / \mathrm{ml})$ were used for saturation binding assay with $\left[{ }^{3} \mathrm{H}\right] \mathrm{NMS}(0.05-1.6 \mathrm{nM}$; PerkinElmer Life and Analytical Science), or competition binding assay $(0.2 \mathrm{nM})$ with the muscarinic antagonist metoctramine (0.1 nM-0.1 mM; Sigma-Aldrich) as previously described (Matucci et al., 2016). Nonspecific binding was defined using $10 \mu \mathrm{M}$ Atropine (Sigma-Aldrich).

\section{Cell Proliferation Assay}

Cell proliferation was determined by MTT assay (Sigma-Aldrich Corp., St. Louis, MO, USA) as previously described (Ambrosini et al., 2015). Cell viability was expressed as relative percentage of viable cells over control, taken as $100 \%$ (mean \pm SEM) from three separate experiments performed in quadruplicate.

\section{In Vivo Study}

\section{Animals}

All animal procedures were carried out according to the EC Directive 86/609/EEC for animal experiments and National guidelines for animal care with the approval of Italian Ministry of Health (Permit Number: 567/2015-PR). Three-month-old, 230-250 g male Wistar rats (Harlan, Milan, Italy) were used. Either saline $(0.9 \%)$ or quisqualic acid (QA; $0.12 \mathrm{M})$ were injected into the right NBM at the following stereotactic coordinates: $A P=-0.2 ; L=-2.8$ and $H=6.8$ from Bregma (Paxinos and Watson, 2006) in anesthetized rats. The animals were equally divided into four groups ( $n=5-6$ per group): Group I, QA-injected and subjected to intravenous administration of human fetal NBM cells (hfNBMs; $1.5 \times 10^{6}$ in $300 \mu \mathrm{l}$ PBS) by the tail vein; Group II, QA-injected; Group III, saline-injected and subjected to intravenous administration of hfNBMs $\left(1.5 \times 10^{6}\right.$ in $\left.300 \mu \mathrm{l} \mathrm{PBS}\right)$; Group IV, un-injected rats (controls). One day prior the intravenous administration of cells and for all the length of the experiment rats were treated with Cyclosporine $(2 \mathrm{mg} / \mathrm{kg} /$ day $)$. Before administration, the cells were labeled with the PKH26 Red Fluorescent dye (Sigma-Aldrich Corp., St. Louis, MO, USA) according to the manufacturer's instructions. Rats from group I were sacrificed on day 1, 7 and 21 after hfNBMs administration. Rats from the other groups were sacrificed on day 21. Anesthetized (chloral hydrate, $400 \mathrm{mg} / \mathrm{kg}$ i.p.) rats were perfused transcardially with $0.9 \%$ saline followed by $4 \%$ paraformaldehyde and brains were paraffin embedded. Livers from rats subjected to intravenous administration of hfNBMs were harvested and analyzed to detect the presence of PKH26 labeled cells in systemic organs.

\section{Immunohistochemistry}

Immunohistochemical analyses of rat brains were performed on $5.0 \mu \mathrm{m}$ coronal paraffin-embedded sections. Anti-GFAP (1:1000; Agilent Technologies, Santa Clara, CA, USA) and anti-ChAT (1:200; Millipore) pAbs were used to detect astrocytes and cholinergic neurons, respectively. ChAT-positive cells in the NBM were counted under a $10 \times$ objective. Five sections per animal, anteroposterior standardized with respect to the injection site and spaced 50-100 $\mu \mathrm{m}$ from one another, were analyzed. The total number of ChAT-positive cells in the QA-injected NBM was averaged, expressed as a percentage of that counted in the saline-injected NBM ( $n=3$ per group), and analyzed using Prism 5.0 (GraphPad Software, San Diego, CA, USA).

\section{Morris Water Maze Test}

During the third week after hfNBMs administration rats were tested in the Morris Water Maze (MWM). The MWM apparatus consisted of a circular pool (1.6 $\mathrm{m}$ in diameter and $0.36 \mathrm{~m}$ high) made of green plastic. The pool was filled to a depth of $20 \mathrm{~cm}$ with water $\left(24-25^{\circ} \mathrm{C}\right)$ that was made dark by the addition of non-toxic dark paint. Rats were tested in the reference memory version of MWM with the procedure previously described for mice (Grossi et al., 2013).

\section{Step-Down Inhibitory Avoidance Task}

The day after the end of MWM task the animals were tested in the Step-Down inhibitory avoidance task as previously described for mouse (Grossi et al., 2009) with some modifications. The apparatus was an open field plexiglas box $(50 \times 25 \times 25 \mathrm{~cm})$ with a steel rod floor and a plexiglas platform $(5 \times 8 \times 25 \mathrm{~cm})$ set on the grid floor to which intermittent electric shocks were delivered.

\section{Statistics}

Data are expressed as mean \pm SEM. Student's paired or unpaired $t$-tests or One- and two-way analysis of variance (ANOVA) followed by Newman-Keuls or Bonferroni post-test analysis were performed, as appropriate, in order to determine statistical significance (set at $p<0.05$ ). Data were analyzed using software package GraphPad Prism (GraphPad Software).

\section{RESULTS}

\section{Phenotypic Characterization of hfNBMs}

In order to isolate human cholinergic neurons from NBM, we dissected and dissociated the corresponding region within the BF of 12-week old human fetuses. The cell suspensions obtained were plated and maintained in Coon's medium supplemented with fetal bovine serum. One week after plating, the cells started to emerge from aggregates and grew as a monolayer population of adherent cells. At this stage, the cells were harvested and replated into fresh medium generating a primary cell culture of hfNBMs neurons (Figure 1A) that was propagated and characterized at different passages.

In order to define the identity of the hfNBM population, we analyzed the expression of immature and mature neural lineage cell markers by qRT-PCR, immunofluorescence analysis and 

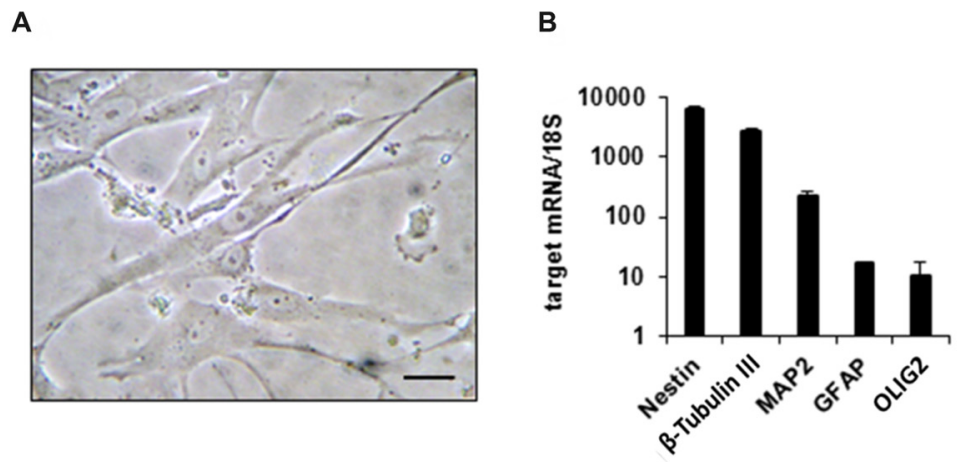

C

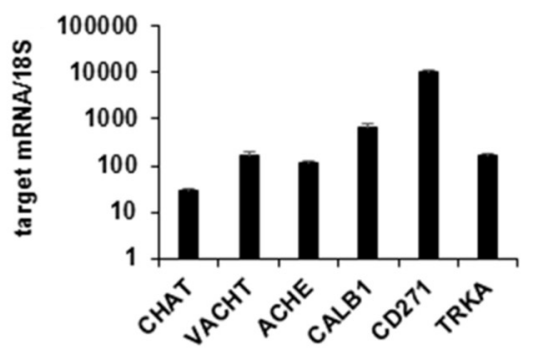

D

E
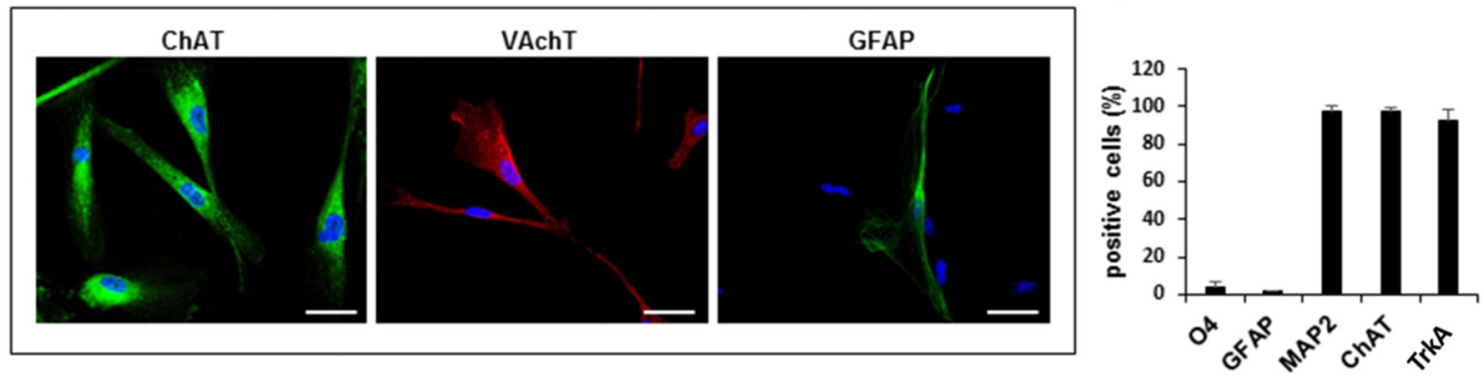

FIGURE 1 | Gene expression profile and phenotypic characterization of hfNBMs. (A) Phase contrast microphotographs showing cell morphology at passage 4 (scale bar $50 \mu \mathrm{m})$. (B,C) Relative mRNA expression by quantitative real time-PCR (qRT-PCR) analysis of target genes normalized over 18S ribosomal RNA subunit, taken as the reference gene; data are mean $\pm \operatorname{SEM}(n=6)$. (D) Representative images of cells expressing ChAT, VAchT and GFAP (DAPI counterstained nuclei, scale bar $50 \mu \mathrm{m})$. (E) Flow cytometric analysis for O4, GFAP, MAP2, ChAT e TrkA proteins; the bar graph shows the percentage of positive cells reported as mean \pm SEM $(n=4)$.

flow cytometry. As shown in Figure 1B, hfNBMs expressed high abundance of both nestin and $\beta$-tubulin III transcripts, which are indicative of neural precursors committed toward the neuronal lineage. Accordingly, we found that the mRNA expression of MAP2, a neuronal marker, was more abundant than the markers of either astroglial (glial fibrillary acidic protein, GFAP) or oligodendroglial (OLIG2) lineages. In addition, hfNBMs expressed a panel of specific genes for NBM cholinergic identity, such as those codifying for enzymes essential for Ach synthesis (choline acetyltransferase, CHAT) and degradation (ACHE), the Ach vescicular transporter (VACHT), the calcium binding protein calbindin 1 (CALB1), and both types of the NGF receptors (TRKA, CD271; Figure 1C). This gene expression profile was retained by cells throughout the different passages in culture (p. 4-p.26).

Immunocytochemical analysis performed in hfNBMs confirmed the prominent neuronal phenotype with a strong immunopositivity to ChAT and VAchT protein expression and a rare detection of GFAP-positive cells (Figure 1D). To provide a quantitative characterization of the hfNBMs phenotype, we used flow cytometry. As shown in Figure 1E, the majority of cells was positive for MAP2 (97.25 $\pm 2.75 \%)$, ChAT $(97.03 \pm 2.09 \%)$ and $\operatorname{TrkA}(92.45 \pm 5.65 \%)$, with a very low percentage of cells being positive for glial markers, such as GFAP $(1.65 \pm 0.05 \%)$ and $\mathrm{O} 4(4.05 \pm 2.55 \%)$. Finally, the cholinergic phenotype of hfNBM cells was confirmed by the detection

of basal release of Ach $(1.23 \pm 0.76 \mathrm{nmol} / \mathrm{ml})$ in the culture medium.

\section{Functional Characterization of hfNBM Cells}

We next profiled the functional features of hfNBMs by studying their electrophysiological properties. The present results have been obtained by patch-clamp whole cell recordings from 102 cells (from p14 to p26). The registered electrophysiological parameters, including resting membrane potential $(\mathrm{Vm})$, membrane resistance $(\mathrm{Rm})$ and membrane capacitance $(\mathrm{Cm})$, showed values typically found in neurons, as reported in Table 1. More relevantly, $89 \%$ of hfNBMs tested (91 out of 102) exhibited fast inward currents in response to a step depolarization (Figure 2A), indicating the expression of functional voltage-gated $\mathrm{Na}^{+}$channels (Table 1). These currents were rapidly activating and inactivating, tetrodotoxin (TTX)-sensitive, and presented an I-V plot

TABLE 1 | Electrophysiological properties of hfNBMs.

Membrane potential (Vm; mV)

Membrane resistance $(\mathrm{Rm} ; \mathrm{M} \Omega)$

Membrane capacitance (Cm; pF)

Sodium current $\left(\mathrm{l}_{\mathrm{Na}}\right)$ amplitude at $0 \mathrm{mV}(\mathrm{pA})$

$\mathrm{K}^{+}$current $\left(\mathrm{I}_{\mathrm{K}}\right)$ amplitude at $+80 \mathrm{mV}(\mathrm{pA})$

Data are presented as mean \pm SEM of $n$ cells. 
A

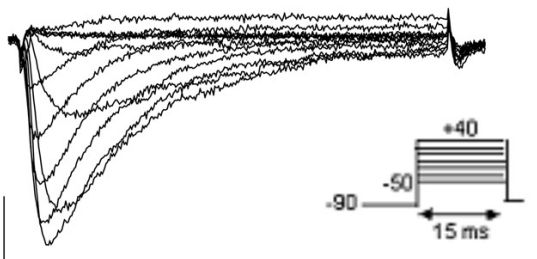

C

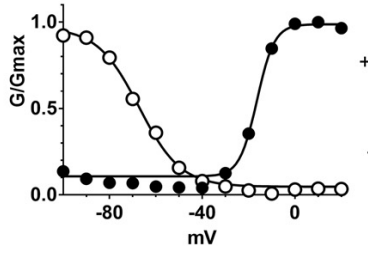

E<smiles>[124IH]</smiles>

G

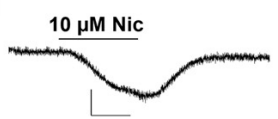

$10 \mu \mathrm{M} \mathrm{MCM}$

K

B

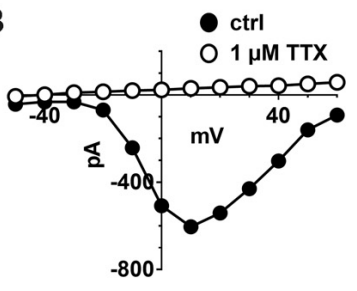

D
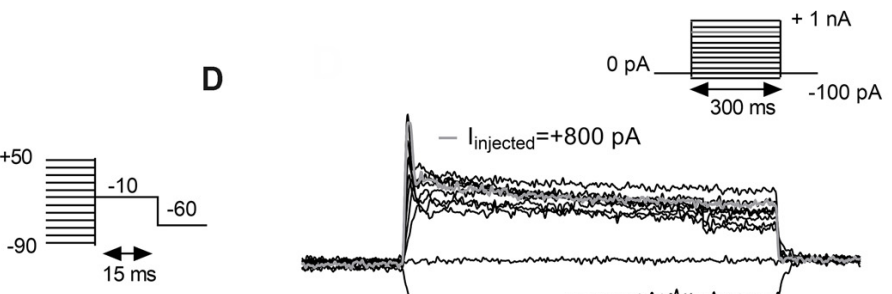

60

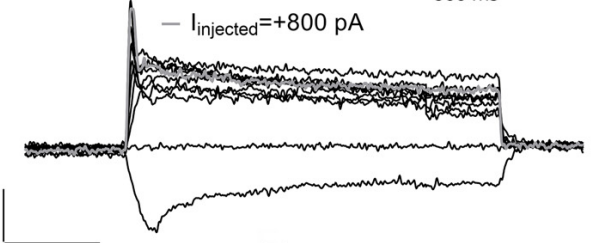

$\mathrm{K}_{\mathrm{A}}$

F

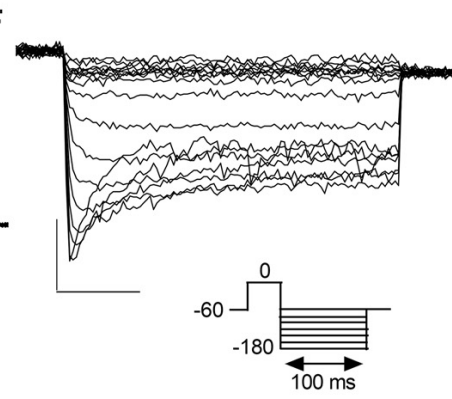

H

I

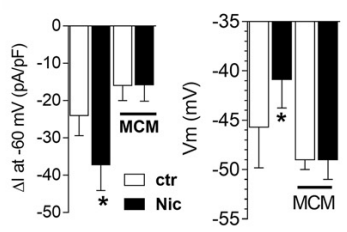

K

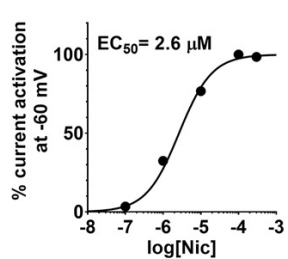

J
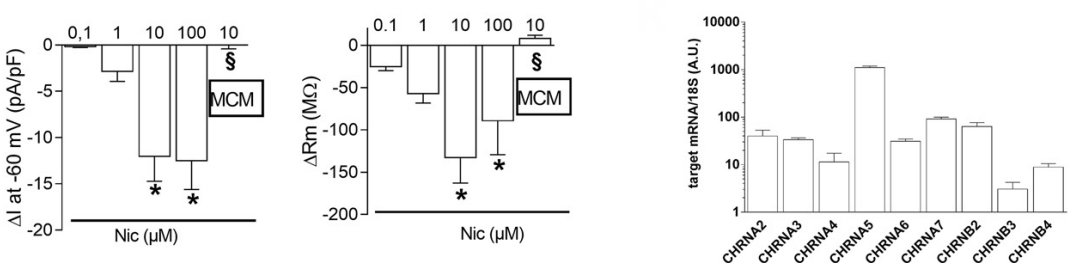

FIGURE 2 | Electrophysiological characterization of hfNBMs. (A) Original $\mathrm{Na}^{+}$currents $\left(\mathrm{I}_{\mathrm{Na}}\right)$ recorded by whole-cell patch clamp technique in a representative cell (at p19). Calibration: 200 pA, 5 ms. (B) Current to voltage relationship (I-V plot) of $I_{\mathrm{Na}}$ recorded in the same cell in absence (•) or in presence (o) of $1 \mu \mathrm{M}$ tetrodotoxin (TTX). (C) Activation (•) and inactivation (o) curves of $I_{\mathrm{Na}}$ evoked in the same cell. Black lines represent fittings with Boltzmann equations $\left(\mathrm{V}_{1 / 2}\right.$ activation $=-14.73 \pm 1.44 \mathrm{mV}$; slope $=4.12 \pm 0.38$; $\mathrm{V}_{1 / 2}$ inactivation $=-56.25 \pm 3.60 \mathrm{mV}$; slope $=5.85 \pm 4.10$ ). (D) Original whole-cell current-clamp recordings in a cell at p19 showing a resting membrane potential of $-48 \mathrm{mV}$. A prominent sag is observed at $-100 \mathrm{pA}$ of current injection. Note that $+800 \mathrm{pA}$ current injection elicited a single, small amplitude, action potential (gray trace). No repetitive firing activity was elicited at higher values of current injection $(+900 \mathrm{pA} ;+1 \mathrm{nA}) . \mathrm{Scal}$ bars: $100 \mathrm{~ms} ; 20 \mathrm{mV}$. (E) Original current traces elicited by a depolarizing voltage step protocol in a representative cell (at p16) expressing only delayed rectifier ( $\mathrm{I}_{\mathrm{K}}$ ) currents (left panel) or in a different cell (at p26) expressing either $I_{K}$ or transient $I_{A}$ currents (right panel). Calibration: 500 pA, 50 ms. (F) Original $K_{i r}$ current traces elicited by a hyperpolarizing voltage step protocol in a representative cell (at p17). Calibration: 200 pA, 25 ms. (G,H) Original current traces (G) and pooled data (H, left panel) recorded in $-60 \mathrm{mV}$ clamped cell where nicotine (Nic, $10 \mu \mathrm{M}$ ) activated an inward current blocked by the selective antagonist Mecamilamine (MCM, $10 \mu \mathrm{M})$. Scale bars: 100 pA, 1 min. Right panel: pooled data indicating that, concomitantly with inward current activation, Nic also concentration-dependently depolarized Vm $\left({ }^{*} p<0.05, n=12\right)$. (I) Concentration-response curve of Nic ( $\left.\mathrm{EC}_{50}=2.6 \mu \mathrm{M}, \mathrm{C} . \mathrm{L} .2 .05-3.4 \mu \mathrm{M}\right)$ in hfNBMs. (J) Pooled data indicating the concentration-dependent effect of Nic, alone or in combination (10 $\mu \mathrm{M}$ Nic) with MCM, on inward current and Rm in hfNBMs. ${ }^{*} p<0.05$ vs. $0.1 \mu \mathrm{M}$ Nic; ${ }^{\S} p<0.05$ vs. $10 \mu \mathrm{M}$ Nic. Data are expressed as mean \pm SEM from at least $n=4$. (K) Relative mRNA expression by qRT-PCR analysis of nicotinic receptor subunits normalized over $18 \mathrm{~S}$ ribosomal RNA subunit, taken as the reference gene, and reported as mean \pm SEM $(n=6)$. 
and an activation-inactivation curve (activation curve: $\mathrm{V}_{1 / 2}=-14.7 \pm 1.4 \mathrm{mV}$; slope factor $=4.2 \pm 0.4$; inactivation curve: $\mathrm{V}_{1 / 2}=-21.7 \pm 6.2$; slope factor $=3.7 \pm 0.2$ ) typical of $\mathrm{Na}^{+}$currents $\left(\mathrm{I}_{\mathrm{Na}}\right)$ recorded in neurons (Figures $2 \mathbf{B}, \mathbf{C}$ ), thus providing functional validation of the neuronal profile of the hfNBMs.

In order to elucidate hfNBM firing activity cells presenting $\mathrm{I}_{\mathrm{Na}}$ currents were investigated under current-clamp configuration. A prominent sag was observed upon negative current injection $(-100 \mathrm{pA}$, Figure 2D) in the majority of cells investigated (see Table 2). A single, small amplitude, action potential (AP) was elicited in some cells upon depolarizing current injections (from $+500 \mathrm{pA}$ ). A modest fast AHP (fAHP) followed the single AP. Repetitive firing was never observed, nor slow AHP (sAHP) at the end of the current step. Quantitative analysis of current-clamp parameters is reported in Table 2.

Concerning investigation of $\mathrm{K}^{+}$currents, we applied a voltage step protocol (Figure 2E, inset) able to activate both slowly activating and not inactivating (delayed rectifier) $\mathrm{K}^{+}$ currents $\left(\mathrm{I}_{\mathrm{K}}\right.$ : Figure $2 \mathrm{E}$, left panel) or fast activating and rapidly inactivating $\mathrm{K}^{+}$currents $\left(\mathrm{I}_{\mathrm{A}}\right.$ : Figure $2 \mathrm{E}$, right panel), in accordance to Sah (1995). Contrarily to $I_{A}$ currents, detected only in a minority of hfNBMs $(37.2 \% ; 38$ out of 102$)$, $\mathrm{I}_{\mathrm{K}}$ currents were observed in all cells tested. We also applied a specific voltage protocol (Figure $2 \mathrm{~F}$, inset) to activate inwardly rectifying $\mathrm{K}^{+}$ currents (Kir), which were recorded in about $40 \%$ of cells ( 43 out of 102; Figure 2F). All these currents $\left(\mathrm{I}_{\mathrm{K}}, \mathrm{I}_{\mathrm{A}}\right.$ and $\mathrm{Kir}$ ) were abolished when extra- and intracellular $\mathrm{K}^{+}$ions were replaced by equimolar $\mathrm{Cs}^{+}$(not shown), thus confirming that they are potassium currents.

We further examined the presence of functional cholinergic (nicotinic and muscarinic) receptors in hfNBMs. First, we investigated the expression of potential nicotinic receptors by applying the selective agonist nicotine (Nic). When a single hfNBM cell was voltage clamped at $-60 \mathrm{mV}$, Nic $(10 \mu \mathrm{M}$, 2 min application) evoked an inward current (Figure 2G, left panel) completely inhibited by the selective Nic antagonist mecamilamine (MCM, $10 \mu \mathrm{M}$, Figure 2G, right panel and Figure 2H, left panel; $p<0.05)$. The effect of Nic was observed in $76.3 \%$ of tested cells (29 out of 38 cells) and was concentration-dependent (Figure 2J, left panel) with $\mathrm{EC}_{50}=2.6 \mu \mathrm{M}$ (confidential limits: 2.05-3.4 $\mu \mathrm{M}$; Figure 2I). Concomitantly with inward current activation, Nic also induced a MCM-sensitive depolarization of Vm (Figure $2 \mathbf{H}$, right panel) and a concentration-dependent decrease in Rm (Figure 2J, right panel).

To explore the functional effects of muscarinic receptors, we applied Ach or carbachol (Cch). A depolarizing voltage-ramp protocol was used to elicit a wide range of overall voltagedependent currents in hfNBMs before, during and after Ach or Cch application. Depolarizing voltage ramps in hfNBMs evoked a majority of $\mathrm{K}^{+}$currents plus a mixture of other voltage-gated currents as indicated by their reversal potential of about $-45 \mathrm{mV}$ (Figure 3A: black trace), which does not coincide with the predicted $\mathrm{K}^{+}$equilibrium potential in our experimental conditions $\left(\mathrm{E}_{\mathrm{K}}=-95.2 \mathrm{mV}\right)$. However, the most of ramp currents are $\mathrm{K}^{+}$currents since they are almost absent in $\mathrm{Cs}^{+}$-replacement experiments (Supplementary Figure S1A). Figure $3 \mathbf{A}$ shows a typical experiment where Ach $(10 \mu \mathrm{M})$ reversibly increased ramp-evoked $\mathrm{K}^{+}$currents in a single cell. The Ach-sensitive current is an outward current with an activation voltage around $-40 \mathrm{mV}$ (Figure 3B). Ach-induced current increase was concentration-dependent (Figure 3C; $p<0.01$ ), prevented by $\mathrm{Cs}^{+}$replacement (Supplementary Figure S1B) and blocked by the muscarinic antagonist atropine (ATR, $100 \mathrm{nM}$, Figure 3C; $p<0.05$ ). The maximal effect of Ach was elicited at a concentration of $10 \mu \mathrm{M}$ with a "bellshaped" curve observed at higher concentrations, probably due to higher rate of Ach degradation by AchE, which is expressed by hfNBMs. Cch mimicked Ach effects on ramp-evoked currents as it concentration-dependently increased outward $\mathrm{K}^{+}$currents (Figure 3D). Finally, either Ach or Cch induced an ATR-sensitive Vm hyperpolarization (Figure 3E; $p<0.001$ ) in line with $\mathrm{K}^{+}$ channel opening. Figures 3F,G show Ach- and Cch-sensitive currents in the absence or presence of ATR, respectively. When tested on the voltage-step protocol to specifically activate $\mathrm{K}^{+}$currents, either Ach (Figure 3K) or Cch (Figure 3M) increased currents with kinetic properties and I-V plot typical of delayed rectifier $\mathrm{I}_{\mathrm{K}}$ conductances (Figures 3K,L, left panels; Figures $\mathbf{3}$ M,N, left panel); both effects were blocked by ATR (Figures 3L,N, right panels). Thus, we conclude that muscarinic receptor activation in hfNBMs by Ach or Cch enhances delayed rectifier $\mathrm{I}_{\mathrm{K}}$ currents.

It should be mentioned that, when Ach was applied in the presence of ATR, we observed a transient increase in the inward component of ramp-evoked currents (Figure $3 \mathbf{H}$ ). This effect, observed only in a subset of hfNBMs (4 out of 7), peaked within the first minute of application and rapidly disappeared before agonist washout (Figure $\mathbf{3 H}$, inset). The reversal potential of Ach-sensitive current recorded in the presence of ATR was around $0 \mathrm{mV}$ (Figure 3I), coherently with the activation of rapidly desensitizing, non-selective cation channels, such as nicotinic receptors.

In order to define which types of cholinergic receptors were expressed by hfNBMs, we analyzed the mRNA expression of the different neuronal subunits of nicotinic receptors (from $\alpha 2$ to $\alpha 7$ and from $\beta 2$ to $\beta 4$ ) along with the different subtypes of

\begin{tabular}{|c|c|c|c|c|c|c|c|}
\hline Passage in culture (p) & n. cells with AP & n. cells with SAG & Sag ratio & AP threshold (mV) & AP amplitude (mV) & AP half width (ms) & fAHP $(\mathrm{mV})$ \\
\hline $\mathrm{p} 15(n=12)$ & $n=4$ & $n=10$ & $2.9 \pm 0.2$ & $-32.2 \pm 1.6$ & $21.8 \pm 2.0$ & $1.95 \pm 0.1$ & $8.01 \pm 3.3$ \\
\hline $\mathrm{p} 19(n=13)$ & $n=3$ & $n=13$ & $3.1 \pm 0.4$ & $-41.1 \pm 2.1$ & $25.5 \pm 4.6$ & $1.06 \pm 0.3$ & $9.3 \pm 3.3$ \\
\hline
\end{tabular}

Data are presented as mean \pm SEM of $n$ cells. 
A

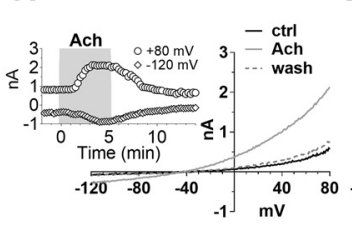

F
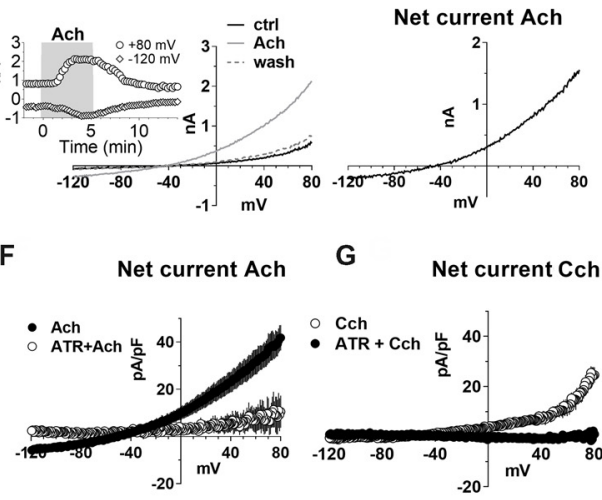

G

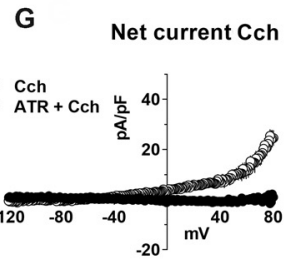

C

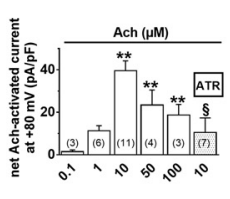

D

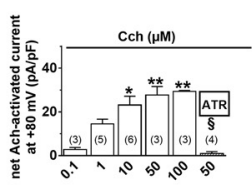

E

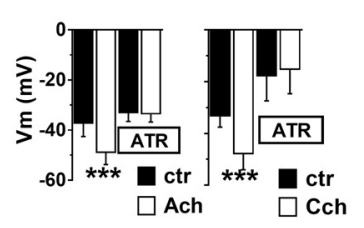

$\mathrm{J}$

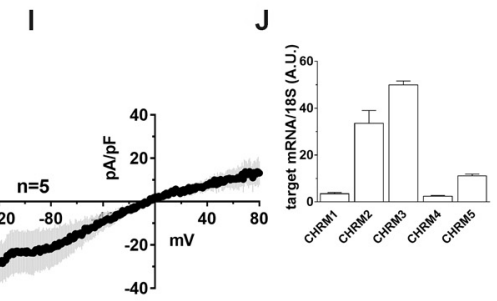

K

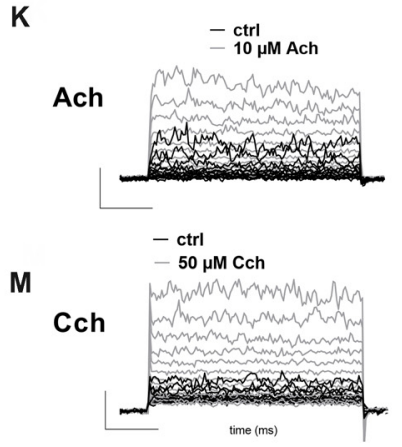

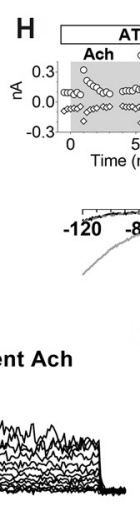
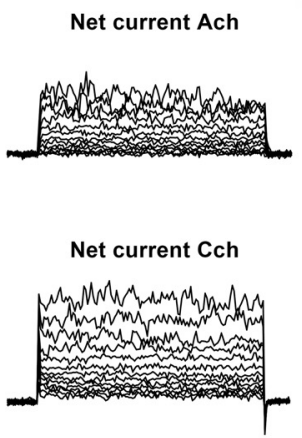

L
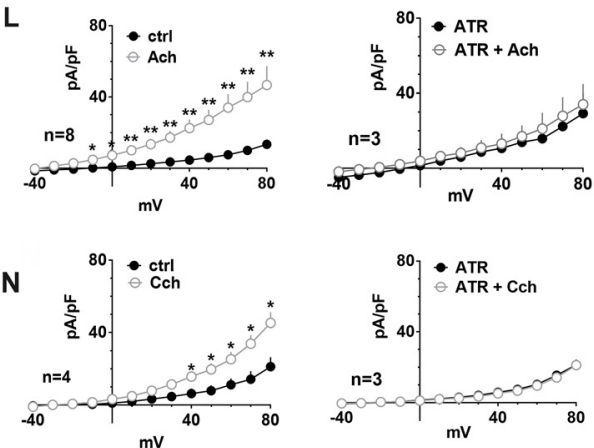

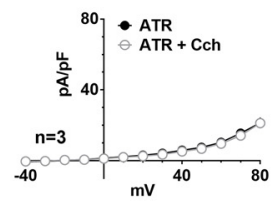

FIGURE 3 | Muscarinic receptor activation by acetylcholine (Ach) or carbachol increases outward $\mathrm{K}^{+}$currents in hfNBMs. (A) Original patch clamp current traces recorded in a representative cell where a voltage ramp protocol (-120/+80 mV, $800 \mathrm{~ms}$ ) was applied before (ctrl), during and after (wash) $10 \mu \mathrm{M}$ Ach application. Inset: time course of ramp-evoked currents at $+80 \mathrm{mV}$ (open circles) or at $-120 \mathrm{mV}$ (open diamonds) in the same cell. (B) Net Ach-sensitive current, obtained by subtraction of the control ramp from that recorded in Ach, in the same cell. (C-E) Pooled data of Ach (C) and Carbachol (Cch, D) concentration-dependent effects on ramp current measured at $+80 \mathrm{mV}$ and on membrane potential $(\mathrm{Vm}, \mathbf{E})$. All these effects induced by muscarinic agonists are blocked by $100 \mathrm{nM}$ atropine (ATR) (C,D) ${ }^{*} p<0.05 ; * * p<0.01,{ }^{* * *} p<0.001$ vs. $0.1 \mu \mathrm{M}$ Ach; ${ }^{\S} p<0.05$ vs. $10 \mu \mathrm{M}$ Ach, One-way analysis of variance (ANOVA), Newman-Keuls post test. Number of cells tested is reported in parenthesis. (E) ${ }^{* * *} p<0.001$ vs. respective ctrl, paired Student's $t$-test, $n=12$ Ach alone; $n=8$ Ach + ATR; $n=6$ Cch alone; $n=4$ Cch + ATR. (F) Averaged Ach-sensitive currents recorded in the absence (filled circles; $n=12$ ) or in the presence (open circles; $n=10$ ) of $100 \mathrm{nM}$ ATR. (G) Averaged Cch-sensitive currents recorded in the absence (filled circles; $n=6$ ) or in the presence (open circles; $n=5$ ) of $100 \mathrm{nM}$ ATR. (H) Original current traces evoked by a voltage ramp protocol in a representative cell in the presence of ATR immediately before (black trace: ctrl) or during (1 min: gray trace; 4 min: dotted gray trace) $10 \mu \mathrm{M}$ Ach application. Inset: Time course of ramp-evoked current at $+80 \mathrm{mV}$ (open circles) or $-120 \mathrm{mV}$ (open diamonds) in the same cell. Note the "nicotinic-like" effect of Ach revealed in the presence of ATR. (I) Averaged net Ach-sensitive current, obtained by subtraction of the control ramp from the Ach trace, recorded in the presence of $100 \mathrm{nM}$ ATR in five cells investigated. (J) Relative mRNA expression by qRT-PCR analysis of muscarinic receptor subunits normalized over $18 \mathrm{~S}$ ribosomal RNA subunit, taken as the reference gene, and reported as mean \pm SEM $(n=6)$. (K) Left panel: original current traces evoked by a voltage step protocol (from -40 to $+80 \mathrm{mV}, 10 \mathrm{mV}$ steps, $200 \mathrm{~ms}$ duration, pre-step $=-80 \mathrm{mV}$ ) in a representative hfNBM cell before (black trace) or during (gray trace) Ach (10 $\mu \mathrm{M}$ ) application. Right panel: net Ach-activated current, obtained by subtraction of the control ramp from the Ach trace, in the same cell. (L) Averaged I-V plots of step-evoked currents recorded in Ach alone $\left(10 \mu \mathrm{M}, n=8\right.$, left panel) or in the presence of ATR (100 nM, $n=3$, right panel). ${ }^{*} p<0.05 ;{ }^{* *} p<0.01$ vs. ctrl; paired Student's $t$-test. (M) Left panel: original current traces evoked by a voltage step protocol in a representative cell before (black trace) or during (gray trace) Cch $(50 \mu \mathrm{M})$ application. Right panel: net Ach-activated current, obtained by subtraction of the control ramp from the Cch trace, in the same cell. (N) Averaged I-V plots of step-evoked currents recorded in Cch alone ( $50 \mu \mathrm{M}, n=4$, left panel) or in the presence of ATR (100 nM, $n=3$, right panel). ${ }^{*} p<0.05$; ${ }^{* *} p<0.01$ vs. ctrl.

muscarinic receptors (from M1 to M5). As shown in Figure 2K, hfNBMs expressed all the nicotinic receptor subunits analyzed, even though at different levels, with $\alpha 5$ subunit (CHRNA5) being the most abundant and $\beta 3$ subunit (CHRNB3) the less expressed (Figure 2K). Similarly, the expression of all the muscarinic receptor isoforms was detected (Figure 3J). In particular, we found high levels of the M2 (CHRM2) and M3 (CHRM3) subtypes. It is known that the M2 isoform is particularly important for cholinergic neuron function as it acts as a pre-synaptic autoreceptor mediating an inhibitory action on Ach release. Hence, the presence of muscarinic receptors, and in particular M2 sites, in hfNBMs was further investigated by radioligand binding analysis. Saturation curves obtained at equilibrium conditions with the non-selective muscarinic antagonist $\left[{ }^{3} \mathrm{H}\right]$-NMS resulted in a maximum binding capacity (Bmax) of $83.52 \pm 18.72 \mathrm{fmol} / \mathrm{mg}$ protein and in a dissociation constant $\left(\mathrm{K}_{\mathrm{D}}\right)$ of $0.07 \pm 0.01 \mathrm{nM}$, thus indicating the presence of high affinity muscarinic cholinergic receptor binding sites (Supplementary Figure S2A). To detect the presence of M2 sites we performed competition binding experiments, using 
A

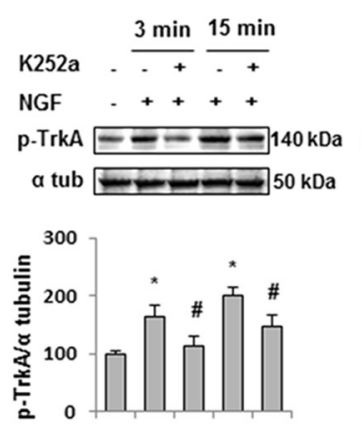

B

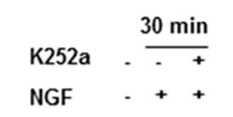

C

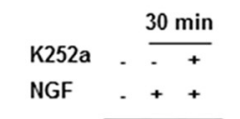

D

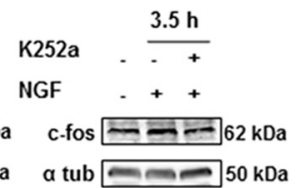

c.fos $\longrightarrow--62 \mathrm{kDa}$
$\mathbf{F}$
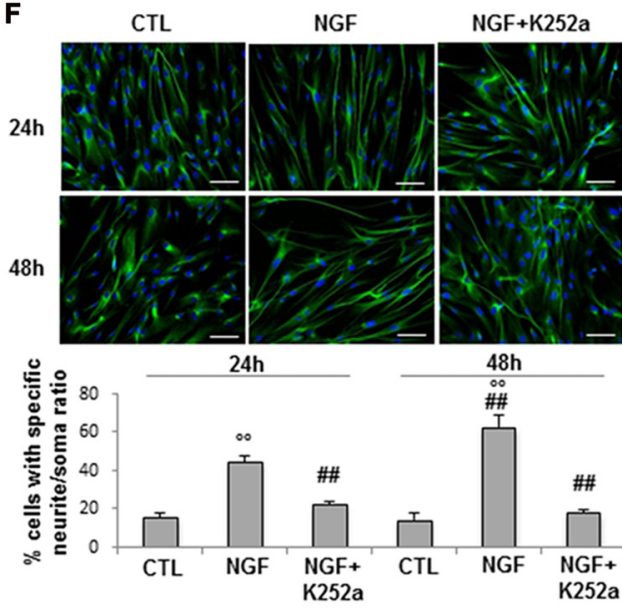

p.CREB $\square 43 \mathrm{kDa}$

$a$ tub $50 \mathrm{kDa}$

$\alpha$ tub $=50 \mathrm{kDa}$
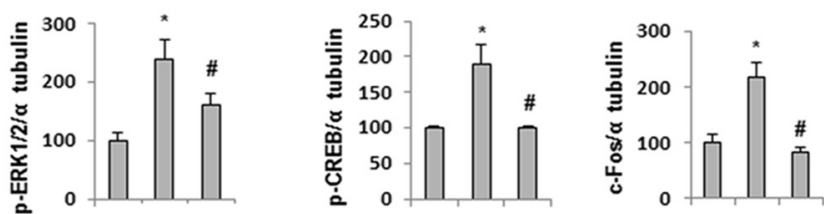

E

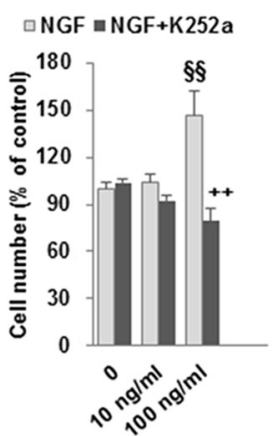

G
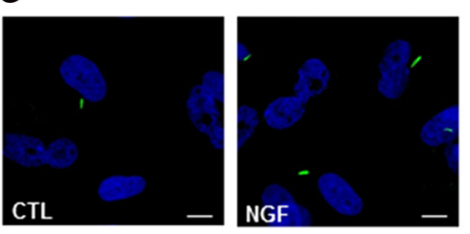

H
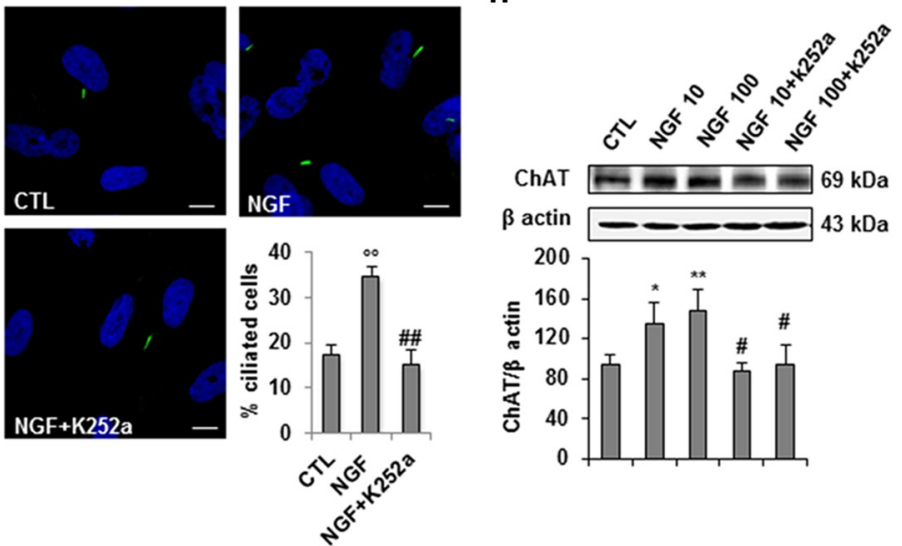

FIGURE 4 | NGF/TrkA signaling activation and effects of NGF on hfNBMs. (A-D) Immunoblotting of TrkA, ERK 1/2 and CREB phosphorylation and c-fos expression induced by $100 \mathrm{ng} / \mathrm{ml} \mathrm{NGF}$ for 3, 15, $30 \mathrm{~min}$ ( $\mathrm{min}$ ) or $3.5 \mathrm{~h}$ in presence or absence of the receptor inhibitor K252a (200 nM); band intensity was normalized over $\alpha$-tubulin signal and expressed as $\%$ of untreated cells; data are mean \pm SEM ( ${ }^{*} p<0.05$ vs. untreated cells, ${ }^{*} p<0.05$ vs. NGF, $n=3$ ). (E) MTT assay of hfNBMs untreated or treated with 10-100 ng/ml NGF for $24 \mathrm{~h}$; data are expressed as percentage of untreated cells and are mean \pm SEM (\$\$ $p<0.005$ vs. untreated cells, ${ }^{++} p<0.005$ vs. NGF, $n=3$ ). (F) Neurite outgrowth analysis by $\alpha$-tubulin staining of hfNBMs treated or not with $100 \mathrm{ng} / \mathrm{ml} \mathrm{NGF}$ for 24 or $48 \mathrm{~h}$ in presence or absence of $\mathrm{K} 252 \mathrm{a}(200 \mathrm{nM})$; the graph shows the percentage of cells with neurites longer than four times the cell body, calculated by counting 10 fields per slide of three separate experiments ( ${ }^{\circ} p<0.001$ vs. CTL, ${ }^{\# \#} p<0.001$ vs. NGF; DAPI counterstained nuclei, scale bar $\left.100 \mu \mathrm{m}\right)$. (G) Acetylated $\alpha$-tubulin staining of primary cilium (green) in hfNBMs (DAPI counterstained nuclei, scale bar $10 \mu \mathrm{m}$ ). The number of ciliated cells was counted in 10 different random fields and expressed as

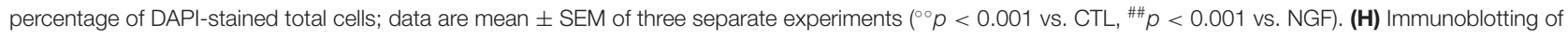
ChAT expression in cells treated or not with 10-100 ng/ml NGF for $24 \mathrm{~h}$ in presence or absence of $200 \mathrm{nM} \mathrm{K252a;} \mathrm{band} \mathrm{intensity} \mathrm{was} \mathrm{normalized} \mathrm{over} \beta$ actin signal and expressed as $\%$ of control; data are mean \pm SEM $\left({ }^{*} p<0.05,{ }^{* *} p<0.01\right.$ vs. CTL; ${ }^{*} p<0.05$ vs. respective NGF; $\left.n=3\right)$.

methoctramine, a muscarinic receptor antagonists that binds to M2 with high affinity. $\left[{ }^{3} \mathrm{H}\right]-\mathrm{NMS}$ binding was completely inhibited by methoctramine providing evidence that M2 is expressed in these cells (Supplementary Figure S2B). The competition-binding assays performed with methoctramine yielded displacement curves, best described with a two-site binding model $\left(\mathrm{pK}_{\mathrm{iH}}=8.18 \pm 0.37 ; \mathrm{pK}_{\mathrm{iL}}=6.21 \pm 0.21 ; n=3\right.$; $p<0.01$; Supplementary Figure S2B).

\section{NGF Activates a Functional TrkA Signaling Pathway in hfNBMs}

Experimental evidence indicates that NGF/TrkA signaling supports survival, maintenance, connectivity and function of the brain cholinergic neurons. As we demonstrated that hfNBMs express the TrkA receptor (Figures 1C,E), we next questioned whether the formation of a NGF-TrkA complex activated a functional TrkA signaling pathway and assessed the phosphorylation of $\operatorname{TrkA}(\mathrm{p} \operatorname{Trk} \mathrm{A})$ and the activation of TrkA down-stream effectors (p-ERK1/2, p-CREB or c-fos). After $24 \mathrm{~h}$ of serum starvation, cells were stimulated with $100 \mathrm{ng} / \mathrm{ml}$ NGF and then harvested at different time points. Western blot analysis with specific antibodies revealed that NGF significantly induced pTrkA within $3^{\prime}-15^{\prime}$ and $\mathrm{p}$-ERK1/2 or $\mathrm{p}$-CREB at 30 , whereas maximum activation of c-fos was within $3.5 \mathrm{~h}$ (Figures 4A-D; $p<0.05$ ). Preincubation with the specific receptor inhibitor K252a prevented all these effects. To test the role of NGF on hfNBMs proliferation, we treated cells with two different concentrations of NGF (10 and $100 \mathrm{ng} / \mathrm{ml}$ ) for $24 \mathrm{~h}$. Using the colorimetric MTT assay we found the $100 \mathrm{ng} / \mathrm{ml}$ NGF caused a significant increase in cell number $(p<0.005$ 

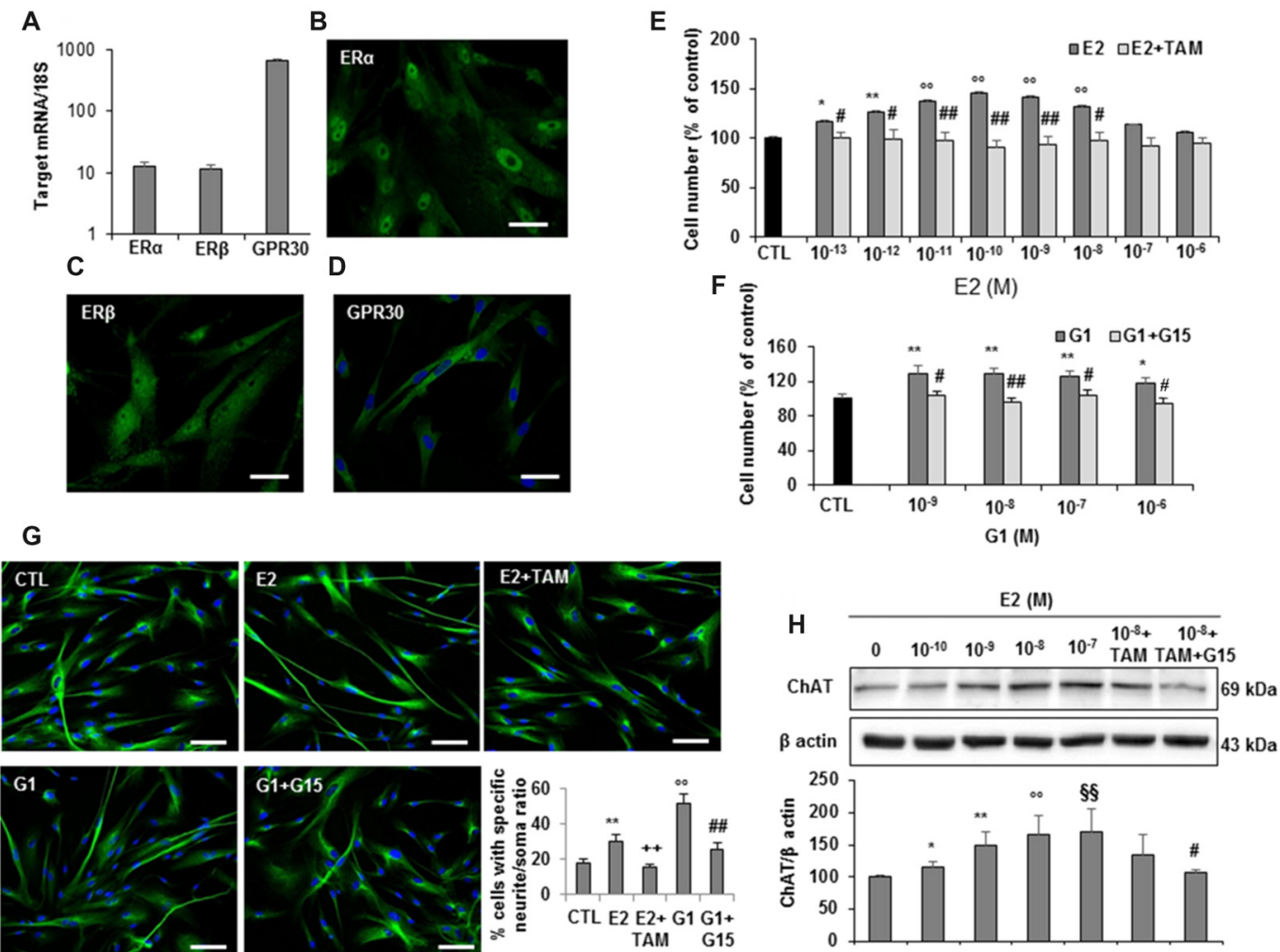

FIGURE 5 | Estrogen receptors expression and estrogen effects on hfNBMs. (A) Relative mRNA expression by qRT-PCR analysis of ER $\alpha$, ER $\beta$ and GPR30 receptors normalized over $18 \mathrm{~S}$ ribosomal RNA subunit, taken as the reference gene, and reported as mean \pm SEM $(n=6)$. (B-D) Immunocytochemical detection of ER $\alpha$, ER $\beta$ and GPR30 in hfNBMs (DAPI counterstained nuclei in (D), scale bar $50 \mu \mathrm{m}$ ). (E,F) MTT analysis of hfNBMs treated or not (CTL) with increasing concentrations of E2 $\left(10^{-13}-10^{-6} \mathrm{M}\right)$ or the GPR30 agonist G1 $\left(10^{-9}-10^{-6} \mathrm{M}\right)$ for $24 \mathrm{~h}$ in the presence or absence of the ER $\alpha / E R \beta$ antagonist tamoxifen (TAM; $\left.100 \mathrm{nM}\right)$ or the GPR30 antagonist G15 $(1 \mu \mathrm{M})$, respectively $\left({ }^{*} p<0.05,{ }^{* *} p<0.01,{ }^{\circ} p<0.001\right.$ vs. CTL; ${ }^{\#} p<0.05,{ }^{\# \#} p<0.001$ vs. treated cells; $\left.n=3\right)$. (G) Neurite outgrowth analysis by $\alpha$-tubulin staining of hfNBMs treated with $10 \mathrm{nM} \mathrm{E2} \mathrm{for} 24 \mathrm{~h}$ in presence or absence of TAM (100 nM) or treated with $100 \mathrm{nM}$ G1 for $24 \mathrm{~h}$ in presence or absence of G15 $(1 \mu \mathrm{M})$; the graph shows the percentage of cells with neurites longer than four times the cell body calculated by counting ten fields per slide of three separate experiments $\left({ }^{* *} p<0.01,{ }^{\circ} p<0.001\right.$ vs. CTL; ${ }^{\# \#} p<0.001,{ }^{++} p<0.005$ vs. treated cells; DAPI counterstained nuclei, scale bar $\left.100 \mu \mathrm{m}\right)$.

(H) Immunoblotting of ChAT expression in serum-starved cells treated or not (CTL) with increasing concentrations of E2 (0.1-100 nM) or with $1 \mathrm{nM}$ E2 in the presence of TAM (100 nM) alone or in combination with G15 $(1 \mu \mathrm{M})$ for $24 \mathrm{~h}$; band intensity was normalized over $\beta$ actin signal and expressed in \% of CTL; data are mean \pm SEM $\left({ }^{*} p<0.05,{ }^{* *} p<0.01,{ }^{\circ} p<0.001,{ }^{\S} \beta p<0.005\right.$ vs. CTL; ${ }^{\#} p<0.05$ vs. E2 $\left.10 \mathrm{nM}, n=3\right)$.

vs. untreated cells), which was blocked by the addition of $\mathrm{K} 252 \mathrm{a}$ (Figure 4E; $p<0.005$ vs. NGF). The effect of NGF on cholinergic neuronal differentiation was determined by analyzing neurite outgrowth. Serum-starved cells were incubated with $100 \mathrm{ng} / \mathrm{ml}$ NGF for 24 and $48 \mathrm{~h}$, and the occurrence of neurite elongation was evaluated by immunofluorescent detection of $\alpha$-tubulin, in comparison with untreated cells (Figure 4F). Neurite outgrowth was determined by measuring the ratio between the longest neurite length and the cell body diameter and calculating the percentage of cells with a particular ratio, as already described (González-Martínez et al., 2004; Sarchielli et al., 2014). As shown in Figure $4 \mathbf{F}$, in the absence of treatments, about $15 \%$ of cells $(15.6 \pm 2 \%$ at $24 \mathrm{~h}$ and $14.3 \pm 3.4 \%$ at $48 \mathrm{~h})$ showed neurites longer than four times the cell body, while the percentage was significantly increased after $100 \mathrm{ng} / \mathrm{ml} \mathrm{NGF}$ treatment at both $24 \mathrm{~h}(44.5 \pm 3.2 \%, p<0.001)$ and $48 \mathrm{~h}(62.8 \pm 6.5 \%$, $p<0.001$ ), an effect prevented by preincubating cells with $\mathrm{K} 252 \mathrm{a}$ at both time points. The effect of NGF on neuritogenesis was confirmed by analyzing growth associated protein 43 (GAP43) protein expression (Supplementary Figure S3). Interestingly, acetylated $\alpha$-tubulin immunostaining, which specifically detects the formation of a primary cilium, revealed that NGF treatment significantly increased the percentage of cells exhibiting this organelle (34.8 $\pm 1.8 \%$; Figure 4G), as compared to untreated cells $(17.3 \pm 2.3 \% ; p<0.0001)$, an effect inhibited by K252a $(15.1 \pm 3.2 \%)$. Because NGF is an essential regulator of cholinergic neuronal phenotype, we also investigated whether the neurotrophin could modulate ChAT expression. As shown in Figure $\mathbf{4 H}$, either 10 or $100 \mathrm{ng} / \mathrm{ml}$ NGF significantly increased ChAT protein expression, compared with untreated cells ( $p<0.05$ and $p<0.01$, respectively), an effect blocked by K252a.

\section{Effects of Estrogen on hfNBMs}

Based on the evidence in literature about the possible association between estrogen plasma levels and $\mathrm{AD}$ incidence, 
A

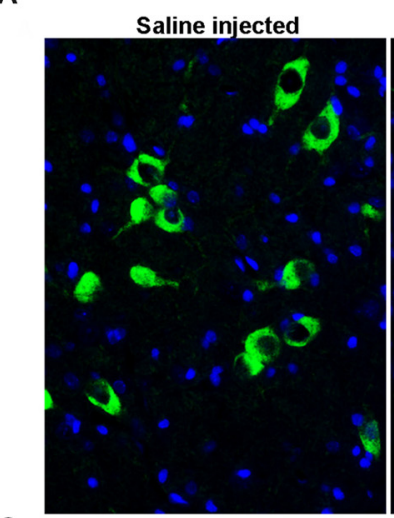

C

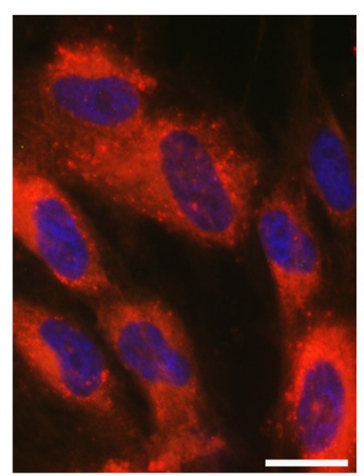

D

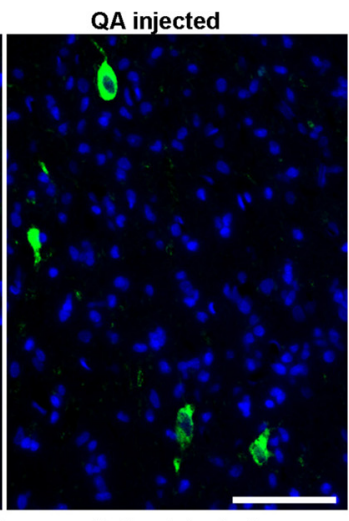

Saline injected

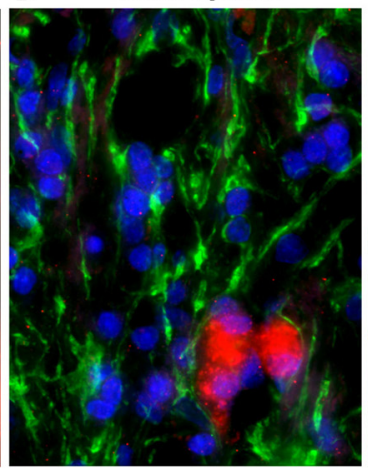

$\mathbf{F}$

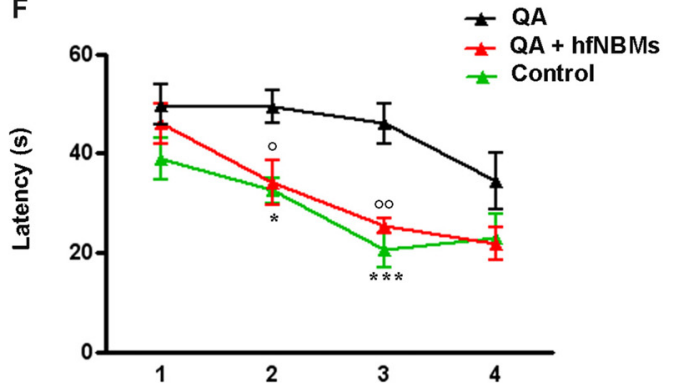

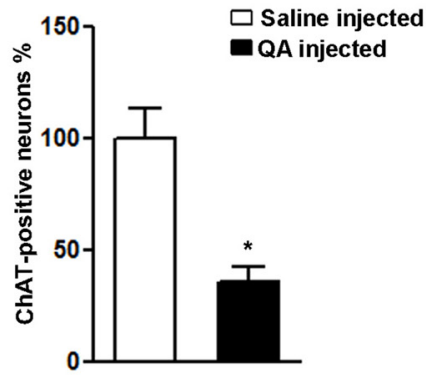

QA injected

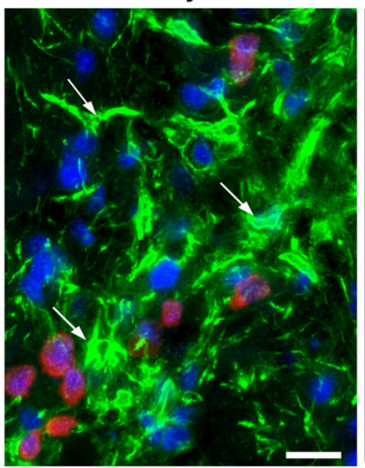

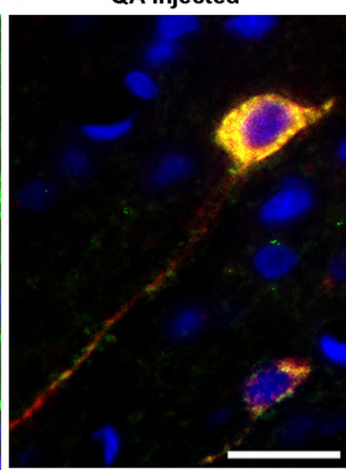

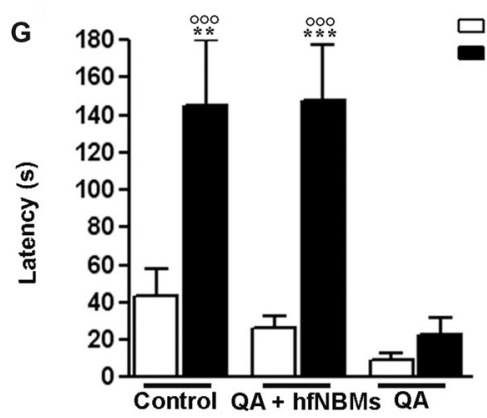

FIGURE 6 | Effects of hfNBMs intravenous administration in nucleus basalis of Meynert (NBM)-lesioned rats. (A,B) Immunohistochemical detection (A) and quantification (B) of ChAT positive cells in the right NBM of quisqualic acid (QA)-injected rats, compared to saline-injected rats; nuclei are counterstained with DAPI (scale bar $50 \mu \mathrm{m})$. Data are expressed as percentage of ChAT-positive neurons (mean $\pm \mathrm{SEM}$ ), taking saline-injected rats as $100 \%$ ( ${ }^{*} p<0.001 ; n=3$ per group). (C) Representative image showing incorporation of the red fluorescent dye PKH26 in hfNBM cells (DAPI counterstaining, scale bar $10 \mu \mathrm{m}$ ). (D) Localization of PKH26-labeled hfNBM cells (red) and evaluation of astrogliosis (white arrows) by anti-GFAP staining (green) in the right NBM of QA-injected compared to saline-injected rats (DAPI counterstaining, scale bar $10 \mu \mathrm{m}$ ). (E) Merge of dual labeling of ChAT (green) and the dye PKH26 (red) in NBM sections of QA-injected rats at day 21 (scale bar $20 \mu \mathrm{m}$ ); ChAT is expressed in hfNBM cells as demonstrated by the yellow color. (F,G) Working memory assessment: (F) Morris water maze (MWM) test, QA-injected rats required significantly more time to find the platform at day 2 and $3\left({ }^{*} p<0.05\right.$, ${ }^{* * *} p<0.001$, respectively) compared to controls, while the QA + hfNBMs group showed a response similar to controls and significantly different from QA rats $\left({ }^{\circ} p<0.05\right.$ day 2 and ${ }^{\circ} p<0.01$ day $3 ; n=5-6$ per group); (G) Step-down inhibitory avoidance test, the training test showed no significant differences between groups. The $24 \mathrm{~h}$ retention test showed increased latencies in controls $\left({ }^{* *} p<0.01\right)$ and $Q A+$ hfNBMs rats $\left({ }^{* * *} p<0.001\right)$ vs. respective training latencies. In $Q A$ rats retention latencies were significantly reduced respect to controls and QA+ hfNBMs $\left({ }^{\circ 00} p<0.001\right)$ and not significantly different from training latencies $(n=5-6$ per group).

we next investigated whether hfNBMs expressed estrogen receptors and responded to $17 \beta$-estradiol (E2). As shown in Figure 5, mRNA expression analysis (Figure 5A) and immunofluorescent detection (Figures 5B-D) demonstrated that hfNBMs possessed both classical estrogen receptors
(ER $\alpha$ and $E R \beta)$, along with the transmembrane $G$ proteincoupled ER (GPR30/GPER1), which is known to mediate rapid non-genomic actions. Increasing concentrations of E2 (0.1-100 nM) or G1 (1-1000 nM), a specific GPR30 agonist, determined a dose-dependent increase in cell number after 
$24 \mathrm{~h}$ exposure, which was reverted by the pre-treatment with either the $\mathrm{ER} \alpha / \mathrm{ER} \beta$ antagonist tamoxifen (100 $\mathrm{nM}$ ) or the selective GPR30 antagonist G15 $(1 \mu \mathrm{M})$, respectively (Figures 5E,F). Similar effects were observed in terms of hfNBMs differentiation, since $24 \mathrm{~h}$ exposure to $10 \mathrm{nM}$ E2 or 100 nM G1 significantly induced neuritogenesis, an effect fully abolished by the respective antagonists tamoxifen or G15 (Figure 5G; $p<0.05)$. In addition, E2 (0.1-100 nM, $24 \mathrm{~h}$ ) determined a significant dose-dependent increase in ChAT protein expression ( $p<0.05$ for each E2 concentration vs. control), which tended to be reduced by tamoxifen pretreatment, although without reaching statistical significance (Figure 5H). Pretreating cells with both G15 and tamoxifen significantly counteracted E2 effect (Figure 5H), thus suggesting a crucial involvement of GPR30 activation in inducing estrogen-mediated ChAT expression. Accordingly, G1 fully mimicked E2 effects on ChAT expression (Supplementary Figure S4).

\section{hfNBMs Improve Memory Functions in NBM-Lesioned Rats}

To test the ability of hfNBMs in improving injured cholinergic function, we performed in vivo experiments using an acute animal model of $\mathrm{AD}$, generated by the unilateral injection of quisqualic acid (QA) into the right NBM of adult male rats. In accordance with previous report (Bartolini et al., 1996), 21 days after NBM lesioning the number of magnocellular ChAT-positive neurons in the QA-injected side was significantly reduced $(-64 \%)$ compared to saline-injected $\mathrm{NBM}$ (Figures 6A,B, $p<0.001$ ). Intravenous administration of hfNBMs was performed in subgroups of QA- and saline-injected rats. To evaluate whether the intravenously administered cells reached the lesioned NBM, hfNBMs were labeled with the red fluorescent dye PKH26 (Figure 6C). As shown in Figure 6D, the immunohistochemical analysis of astrogliosis revealed the presence of reactive GFAP-positive astrocytes with enlarged cell bodies and long processes in NBM sections from QA- but not saline-injected rats (Figure 6D). The presence of PKH26labeled hfNBMs was detected both in the QA- and saline-injected NBM (Figure 6D). Immunofluorescent staining with ChAT antibody of NBM sections from the experimental rats showed that PKH26-labeled cells were ChAT-positive, as exemplified by the merge image from QA-injected rats at day 21 (Figure 6E). No PKH26-labeled cells were detected in uninjured brain areas, including cortex, striatum, medial septum and peduncolopontine nuclei of both cerebral hemispheres, nor in the left unlesioned NBM.

The effects of hfNBMs administration on cognitive functions were assessed with both MWM and step down inhibitory avoidance tests. At day 21, rats from each experimental group ( $n=5-6$ per group) were trained for 4 days in the MWM task to learn where the hidden platform was located. The rats were naive to the water maze and showed no deficiencies in swimming abilities, directional swimming towards the platform, or climbing onto a hidden platform during training trials. As shown in Figure 6F, QA-injected rats required significantly more time to find the platform compared to controls $(p<0.05$, day 2 , and $p<0.001$, day 3$)$. Interestingly, rats from the QA-injected + hfNBMs group were good swimmers and showed an appropriate swim-search response after being placed in water, significantly shortening the escape latency during the 2- and 3-day acquisition phase compared to QA-injected group (Figure 6F; $p<0.05$, day 2 and $p<0.01$, day 3 ). At day 4 of acquisition phase, QA-injected rats shortened the escape latency with no significant differences when compared to QA+ hfNBMs or control rats (Figure 6F). No differences were detected in the escape latency in all acquisition phase sessions between control and saline-injected + hfNBMs rats (data not shown). The same animals were then tested for the step-down inhibitory avoidance ( $n=5-6$ per group; Figure 6G). No significant differences were observed among all groups during the training test. In the $24 \mathrm{~h}$ retention test step-down latencies recorded for controls and QA+ hfNBMs rats were significantly increased $(p<0.01$ and $p<0.001$ vs. their respective training latencies). In QA rats retention latencies were significantly reduced compared to controls and QA+ hfNBMs $(p<0.001)$ and not significantly different from training latency, indicating that QA rats were unable to memorize the punishment and to perform the inhibitory avoidance. Administration of hfNBMs to QA rats significantly improved their performance, which reached the level displayed by control rats (Figure 6G). No differences were detected during retention test between control and saline injected + hfNBMs rats (data not shown).

\section{DISCUSSION}

The impairment of the BF cholinergic system, especially NBM, is a crucial event in the progression of memory and cognitive decline associated to neurodegenerative disorders, such as AD. It therefore represents the main target for therapeutic approaches (Ferreira-Vieira et al., 2016). The most efficient treatment should be able to direct a regenerative process of the damaged brain areas, a possibility currently under consideration in the attempt to establish efficient stem cell-based replacement therapies for AD. Studies on derivation of functional BF cholinergic neurons from stem cells are limited (Bissonnette et al., 2011; Liu et al., 2013; Yue et al., 2015) and, although the regenerative potential has been proved in $\mathrm{AD}$ animal models, the actual application in clinical protocols seems far to be reached. Besides the generation of functionally integrated stem cell-derived cholinergic neurons, major efforts should be directed towards unraveling those mechanisms that may protect NBM neurons from degenerative insults, with the main aim of identifying efficient strategies to prevent $\mathrm{AD}$ pathogenesis. In this study, we provide for the first time a comprehensive phenotypic and functional characterization of primary human cholinergic neurons isolated from the fetal NBM. They may represent not only an optimal resource to study developmental processes of neurons already committed towards the NBM cholinergic phenotype, but also a useful model in the assessment of regenerative protocols based on an efficient generation of stem cell-derived NBM cholinergic neurons. Indeed, we show that hfNBMs, without any manipulation and via systemic injection, 
were able to determine a functional recovery in NBM-lesioned rat brain.

The hfNBM primary cultures clearly showed a prominent neuronal phenotype, with high levels of MAP2 transcripts, which was confirmed by the flow cytometry detection of almost $100 \%$ of MAP2-positive cells. In addition, hfNBMs abundantly express both the neural precursor marker nestin and the neuronal progenitor marker $\beta$ tubulin III, indicating coexisting undifferentiated cells already committed towards a specific phenotype. This plasticity was expected in primary cultures of fetal origin (Sarchielli et al., 2014) and may be of great value to study mechanisms potentially interfering with cholinergic neuron maturation and function. The hfNBM identity as cholinergic neurons was demonstrated by the expression of the major components of the cholinergic system, including proteins important for Ach synthesis, transport and hydrolysis (ChAT, VAchT and AchE, respectively). Accordingly, a basal release of Ach by these cells has been detected. In addition, the hfNBM neurons express CALB1 along with both high- (TrkA) and low- (p75/CD271) affinity NGF receptors, which altogether define the regional specificity of NBM cholinergic neurons (Abreu-Villaça et al., 2011). Indeed, in humans all the BF cholinergic neurons were also CALB1-positive (Geula et al., 1993) and co-expression of CALB1 with both NGF receptors is consistent with the pattern described for the Ch4 neurons of the human brain, which correspond to NBM according to the Mesulam's nomenclature (Mesulam, 2013). Although, we might expect to also isolate GABAergic and glutamatergic neurons from the $\mathrm{BF}$, in our primary cultures the majority of cells, as detected by flow cytometry, were ChAT-positive (97\%). Our findings are in accordance with previous immunocytochemical and hybridization studies (Mufson et al., 2003) reporting a high enrichment of cholinergic neurons (90\%) within the human NBM/Ch4 region.

The functional validation of the neuronal profile of hfNBM cells was provided by electrophysiological measurements, which demonstrated the presence of a polarized membrane potential $(\sim-45 \mathrm{mV})$, the expression of functional, TTX-sensitive, voltage-gated $\mathrm{Na}^{+}$channels $\left(\mathrm{I}_{\mathrm{Na}}\right)$, along with the presence of active phenomena of firing activity, in line with previous data on human developing neuronal cells (Lepski et al., 2011; Song et al., 2013). Furthermore, we detected transient $I_{A}$ currents, delayed rectifier $I_{K}$ and inwardly rectifying $K_{i r}$ currents. The presence of $\mathrm{I}_{\mathrm{A}}$ and $\mathrm{I}_{\mathrm{K}}$ currents has been previously detected in human fetal brain neurons (Sah, 1995). In our population of isolated hfNBM neurons, $\mathrm{I}_{\mathrm{K}}$ were the predominant conductance recorded in all cells tested and $\mathrm{I}_{\mathrm{Na}}$ currents were present in the vast majority $(89 \%)$ of hfNBMs. Transient $\mathrm{I}_{A}$ currents and Kir conductances were only detected in a subset of recorded cells ( $37 \%$ and $41 \%$, respectively), suggesting that hfNBMs culture might include a heterogeneous population of neurons at different maturation steps or with distinct arrays of $\mathrm{K}^{+}$channels. Interestingly, hfNBMs also exhibited the presence of functional cholinergic receptors. Ach binds to two distinct types of receptors: ionotropic nicotinic (nAchR) and metabotropic muscarinic (mAchR) receptors, located in both pre- and post-synaptic membranes of neurons. In particular, $\mathrm{nAchR}$ are pentameric ligand-gated ion channels resulting from the combination of different $\alpha(\alpha 2-10)$ and $\beta(\beta 2-4)$ subunits (Gotti et al., 2007). We demonstrated not only that hfNBM cells expressed, at mRNA level, all nAchR subunits investigated, with the $\alpha 5$ subunit being the most abundant, but also that they were able to respond to nicotine by triggering a $0 \mathrm{mV}$-reverting ramp-evoked current and membrane depolarization. Our results are consistent with the early detection of $\mathrm{nAChR}$ proteins and gene transcripts in brains from human fetuses of 4-12 weeks of gestational age (Hellström-Lindahl et al., 1998). They also further support the important role proposed for nAchRs during brain development in modulating dendritic outgrowth, establishment of neuronal connections and synaptogenesis (Abreu-Villaça et al., 2011). Future investigations aimed at identifying the specific subunits assembly of nAchRs, which are active in hfNBMs in the different maturation states, will help to clarify the neurodevelopmental actions of Ach. The relative abundancy of the $\alpha 5$ subunit in hfNBMs may suggest the importance of this subunit in regulating the nAchRs activity during cholinergic neuron development. Indeed, among nAchRs subunits, the $\alpha 5$ plays a peculiar functional role, since it contributes to the lining of functionally unique nAchR channels, but only if co-expressed with both another alpha- and beta-type subunit (Ramirez-Latorre et al., 1996). Moreover, studies in animal models have shown that ubiquitously distributed presynaptic nAchRs serve as ligandgated ion channels that promote neurotransmitter release (Wonnacott et al., 2006). Hence, further studies should be addressed to clarify if $\mathrm{nAchRs}$ in our cellular model of cholinergic neurons could play a role as presynaptic receptors recruited to regulate Ach release.

We also clearly show that Ach activates mAchRs receptors in hfNBMs. Five subtypes of mAchRs have been described (M1-M5) with an inhibitory (M2 and M4) or excitatory (M1, M3 and M5) action on neuronal excitability (Caulfield, 1993). M2 and M3 subtypes resulted the most abundant in hfNBMs. Again, our results are consistent with literature data highlighting the importance of mAchRs, like nAchRs, in the developing central nervous system linked to clear effects of Ach on neuronal proliferation, differentiation and survival (Abreu-Villaça et al., 2011). Moreover, the presence of abundant M2 transcripts along with specific M2-type binding sites may reflect a prominent regulatory role of $\mathrm{mAchRs}$ as autoreceptors on Ach release in hfNBM cells. In line with this observation, we describe an ATR-sensitive increase in delayed rectifier outward $\mathrm{I}_{\mathrm{K}}$ currents upon Ach or Cch application, as known for Gi coupled metabotropic receptors such as M2 and M4 subtypes (Caulfield and Birdsall, 1998). This effect could be either due to a direct action of the $\mathrm{G}$ protein subunit/s on $\mathrm{K}^{+}$channels, or by second messenger-mediated (i.e., cAMP-mediated) channel modulation, as both possibilities have been described in other cellular systems (Inanobe and Kurachi, 2014). Since either Ach or Cch effects on $\mathrm{I}_{\mathrm{K}}$ currents peaked within only 1-2 min from agonist superfusion, we may hypothesize a direct action of the $G_{\alpha}$ or $G_{\beta / \gamma}$ subunit/s coupled to muscarinic receptor activation on delayed rectifier $\mathrm{K}^{+}$channels. Overall, it emerges 
from present data that nicotinic receptors excite hfNBMs by depolarizing the cell membrane and approaching them to firing threshold, whereas mAchR activation, by opening $\mathrm{I}_{\mathrm{K}}$ channels, could result inhibitory on neuronal excitability. However, given the fetal origin of our cellular model, the specific recruitment of either nicotinic or muscarinic receptor subtypes could be related to maturation processes of the cholinergic phenotype induced by Ach, as reported (BruelJungerman et al., 2011), rather than to specific functional responses, such as those occurring in the context of adult brain circuits. This issue merits further investigations in future studies.

Several lines of evidence indicate that NGF is an endogenous neurotrophic factor crucially required to support survival, maintenance, connectivity and function of the brain cholinergic neurons, as derived by extensive research involving a wide range of animal models (Dekker et al., 1991; Koliatsos et al., 1991). Consistently with these findings, we demonstrated that NGF induces, via TrkA activation and its downstream signaling pathways, proliferation, neuritogenesis and ChAT expression in hfNBMs, thus confirming also in a human model the role played by the neurotrophin in the development and maintenance of the cholinergic phenotype. Accordingly, phase 1 clinical trials based on NGF gene delivery to NBM proved that this approach has the potential to improve cognitive symptoms and modify neurological disease progression in $\mathrm{AD}$ patients (Tuszynski et al., 2005). Interestingly, among the effects observed upon NGF treatment, the increased percentage of hfNBMs exhibiting a primary cilium appears of particular interest given the recent involvement of this organelle in the adult neurogenesis (Han et al., 2008). Primary cilia are non-motile, $4-8 \mu \mathrm{m}$ long sensory antennas protruding from the surface of nearly all cells of the body, able to mediate the cellular response to extracellular signals growth and other factors (Armato et al., 2013). In particular, primary cilia have been detected in adult murine and rat neural stem/progenitor cells of the dentate gyrus's subgranular zone (SGZ), which may produce new granule cells when they are needed for new memory encoding (Einstein et al., 2010). Accordingly, primary cilia of SGZ cells showed structural damages in transgenic AD mouse models (Rodríguez et al., 2008). Nevertheless, the precise role that primary cilia play in the overall neuronal function is still unknown. Our results strongly suggest for the first time that primary cilia may be involved in the NGF-driven maturation of human NBM cholinergic neurons. Further investigations may clarify the effective role of the primary cilium in the signaling mechanisms directly or indirectly recruited by NGF in the overall neuronal maturation. Since most evidence suggested that primary cilia signaling is based on an enrichment of receptors on its membrane (Händel et al., 1999), it would be interesting to analyze whether NGF receptors co-localize to primary cilia of hfNBMs. In this regard, the p75 low-affinity NGF receptor has been found in adult murine hippocampal dentate gyrus granule cells (Chakravarthy et al., 2010) and showed to be involved in the amyloid $\beta_{42}$ toxic activity (Perini et al., 2002).

Accumulating evidence in animal models indicated that various neuronal functions, including learning and memory processes, are beneficially influenced by estrogens (EnglerChiurazzi et al., 2016). Accordingly, in humans, it has been reported that the risk of $\mathrm{AD}$ is higher for women than men, especially in post-menopausal women compared to their male counterpart, clearly suggesting a neuroprotective role of estrogens (Breitner et al., 1988; Jorm and Jolley, 1998). Indeed, a recent meta-analysis of modifiable risk factors for $\mathrm{AD}$ identified the use of estrogens among the protective factors (Xu et al., 2015). Although controversial literature exists about the effectiveness of estrogen replacement therapy in preventing $\mathrm{AD}$, several animal studies have shown that estrogens are neuroprotective for multiple targets in the central nervous system, including hippocampal neurogenesis and BF cholinergic neuron survival and function (Engler-Chiurazzi et al., 2016). In this regard, we report that hfNBMs express estrogen receptors. In particular, besides the classical nuclear $\operatorname{ER} \alpha$ and $\operatorname{ER} \beta$, hfNBM cells also express the membrane-associated GPR30, a G protein-coupled receptor responsible for the rapid non-genomic actions of estrogens (Revankar et al., 2005). The most striking finding of our study is that the different types of estrogen receptors could be differently involved in mediating estrogenic action on growth, differentiation and maintaining of cholinergic phenotype in hfNBMs. Indeed, exposing cells to E2 induced cell proliferation, neuritogenesis and ChAT expression, with all effects mimicked by the exposure to G1, the selective GPR30 agonist, clearly implying the recruitment of rapid non-genomic mechanisms. However, pre-treatment with tamoxifen (an effective ER $\alpha$ and ER $\beta$ antagonist) counteracted all the estrogen-induced changes in hfNBMs, except for ChAT protein expression increase that was significantly prevented only in the presence of the specific GPR30 antagonist G15. Hence, GPR30 appears to be crucially required for the estrogenic action on maintenance of cholinergic phenotype in hfNBMs. Our findings are in good agreement with the recent evidences in experimental animals, which revealed that GPR30 is positioned on BF cholinergic neurons to mediate important estrogenic actions, such as effects on cognitive performance (Hammond and Gibbs, 2011) and Ach release in the hippocampus (Gibbs et al., 2014). A better understanding of the precise molecular mechanisms through which GPR30 regulates memory and cognition in humans will help to the development of more effective therapies for preventing and treating cognitive decline associated with $\mathrm{AD}$.

Another important finding of our study is the demonstration that hfNBMs were able to improve cognitive functions when administered to a rat model of $\mathrm{AD}$, obtained via the unilateral lesion of NBM. In particular, hfNBMs administration in NBM-lesioned rats determined a significant recovery of memory deficits. Memory is the product of dynamic interactions among multiple systems in the brain. Notably, working and spatial memory for the platform location appears to be mainly hippocampus-dependent as this brain area, which receives cholinergic inputs from the BF nuclei, is necessary for acquisition and retrieval of spatial information. Indeed, lesions of the medial septal and diagonal band cholinergic neurons result in deficits in the MWM task (Janis et al., 1998). However, memory performance in MWM is also affected by the NBM neuron activity resulting NBM lesion and stimulation in deficits and 
improvements, respectively, in the acquisition of spatial memory tasks (Lee et al., 2016). The step-down inhibitory avoidance memory depends mainly on the integrated activity of entorhinal, parietal cortex and CAI areas. Hence, lesions of the NBM, which provides the major cholinergic innervation to the entire cortical mantle, are mainly responsible for memory deficits in this task (Torres et al., 1994; Giovannini et al., 2015; Lee et al., 2016). Accordingly, our findings showing that spatial memory impairments in the MWM test of untreated NBM-lesioned animals recovered after 3 days of training indicate that NBM does not play a primary role in the acquisition of spatial memory task and that other cellular and molecular mechanisms underlie the spontaneous and hfNBM-induced spatial memory restoration. In contrast, memory deficits in the step-down inhibitory avoidance test of untreated NBM-lesioned animals persisted during the entire test, indicating that the memory disturbance in this task mainly reflects NBM activity.

We did not specifically analyze whether neuronal connections between donor and host neurons occurred, as well as no data about an improvement of cholinergic activity (Ach secretion) in brains from QA-lesioned/hfNBM-treated compared to QAlesioned/untreated rats are provided. However, based on both the in vitro functional characterization and in vivo results, it is plausible that hfNBMs, at least for 3 weeks, retained their phenotype able to exert a trophic activity on the host injured cholinergic area. In addition, the fact that hfNBMs, intravenous administered, were detected in the NBM injected either with QA or saline and not in other brain areas not interested by the insult indicates that a damage of the blood brain barrier favors the migration of cells to injured brain sites. Although the manner in which injected neurons systemically reach the damaged brain area remains unknown, similar studies in models of neurodegenerative diseases demonstrated that intravenous route for stem cell transplantation is feasible and effective (Lee et al., 2005; Shen et al., 2010; Martínez-Morales et al., 2013). Most likely, the ability of migrating and penetrating to a specific site, as well as recapitulating specific functions, is related to an already committed phenotype and may be ascribed to the fetal origin of our cellular model, thus supporting the validity of such a model for cell-based therapy purposes. Indeed, as occurred for the assessment of cell-based therapy protocols for the treatment of PD (Grealish et al., 2014), human fetal neurons may serve as an important reference in terms of morphology, maturation, marker expression and overall phenotypic properties.

\section{REFERENCES}

Abreu-Villaça, Y., Filgueiras, C. C., and Manhães, A. C. (2011). Developmental aspects of the cholinergic system. Behav. Brain Res. 221, 367-378. doi: 10.1016/j.bbr.2009.12.049

Ambrosini, S., Sarchielli, E., Comeglio, P., Porfirio, B., Gallina, P., Morelli, A., et al. (2015). Fibroblast growth factor and endothelin-1 receptors mediate the response of human striatal precursor cells to hypoxia. Neuroscience 289, 123-133. doi: 10.1016/j.neuroscience.2014.12.073

Armato, U., Chakravarthy, B., Pacchiana, R., and Whitfield, J. F. (2013). Alzheimer's disease: an update of the roles of receptors, astrocytes and
In conclusion, this study is the first in which human NBM cholinergic neurons are isolated and fully characterized and where their potential role for functional repair, without any manipulation, has been tested in vivo in an experimental model of AD. Such a model of young neurons, clearly programmed to the cholinergic function, possesses functional channels and receptors able to respond to important physiological regulators (Ach, NGF, estrogens). It therefore represents an excellent tool to study the ontogenetic mechanisms regulating the development and maintenance of the human BF cholinergic system. In addition, this cellular model could be useful to assess new lines of research including disease modeling, cell-based therapy and drug screening in the field of neurodegenerative disorders.

\section{AUTHOR CONTRIBUTIONS}

AM conceived and carried out experiments, interpreted the results and wrote the manuscript. ES and GG contributed to the cell culture isolation, characterization and in vitro experiments. EC and AMP performed the electrophysiological analysis. DP, $\mathrm{PN}$ and FC contributed to the in vivo experiments and performed behavioral tests. PC contributed to gene expression experiments. LB and BM contributed to cell phenotype characterization. $\mathrm{SA}$ and GC contributed to the in vitro experiments, $\mathrm{RM}$ and $\mathrm{RV}$ performed binding experiments. SB contributed to the fetal human tissue collection. MM and PG contributed to the interpretation of the results and revised the manuscript. GBV contributed to the conception of the project and the interpretation of results, and wrote the manuscript. All authors have approved the final version of this manuscript, agree to be accountable for all aspects of the work and qualify for authorship.

\section{ACKNOWLEDGMENTS}

This work was supported by a grant from University of Florence. We would like to thank Dr. Leonardo Dini for his helpful assistance and suggestions regarding current-clamp experiments.

\section{SUPPLEMENTARY MATERIAL}

The Supplementary Material for this article can be found online at: https://www.frontiersin.org/articles/10.3389/fncel. 2017.00339/full\#supplementary-material

primary cilia (review). Int. J. Mol. Med. 31, 3-10. doi: 10.3892/ijmm. 2012.1162

Bartolini, L., Casamenti, F., and Pepeu, G. (1996). Aniracetam restores object recognition impaired by age, scopolamine, and nucleus basalis lesions. Pharmacol. Biochem. Behav. 53, 277-283. doi: 10.1016/0091-3057(95)02021-7

Bissonnette, C. J., Lyass, L., Bhattacharyya, B. J., Belmadani, A., Miller, R. J., and Kessler, J. A. (2011). The controlled generation of functional basal forebrain cholinergic neurons from human embryonic stem cells. Stem Cells 29, 802-811. doi: $10.1002 /$ stem. 626

Björklund, A., and Lindvall, O. (2000). Cell replacement therapies for central nervous system disorders. Nat. Neurosci. 3, 537-544. doi: 10.1038/75705 
Breitner, J. C., Silverman, J. M., Mohs, R. C., and Davis, K. L. (1988). Familial aggregation in Alzheimer's disease: comparison of risk among relatives of early-and late-onset cases and among male and female relatives in successive generations. Neurology 38, 207-212. doi: 10.1212/WNL.38.2.207

Bruel-Jungerman, E., Lucassen, P. J., and Francis, F. (2011). Cholinergic influences on cortical development and adult neurogenesis. Behav. Brain Res. 221, 379-388. doi: 10.1016/j.bbr.2011.01.021

Caulfield, M. P. (1993). Muscarinic receptors-characterization, coupling and function. Pharmacol. Ther. 58, 319-379. doi: 10.1016/0163-7258(93) 90027-b

Caulfield, M. P., and Birdsall, N. J. (1998). International Union of Pharmacology. XVII. Classification of muscarinic acetylcholine receptors. Pharmacol. Rev. 50, 279-290.

Chakravarthy, B., Gaudet, C., Ménard, M., Atkinson, T., Chiarini, A., Dal Prà, I., et al. (2010). The p75 neurotrophin receptor is localized to primary cilia in adult murine hippocampal dentate gyrus granule cells. Biochem. Biophys. Res. Commun. 401, 458-462. doi: 10.1016/j.bbrc.2010.09.081

Coppi, E., Pedata, F., and Gibb, A. J. (2012). P2Y $Y_{1}$ receptor modulation of $\mathrm{Ca}^{2+}$. activated $\mathrm{K}^{+}$currents in medium-sized neurons from neonatal rat striatal slices. J. Neurophysiol. 107, 1009-1021. doi: 10.1152/jn.00816.2009

Dekker, A. J., Langdon, D. J., Gage, F. H., and Thal, L. J. (1991). NGF increases cortical acetylcholine release in rats with lesions of the nucleus basalis. Neuroreport 2, 577-580. doi: 10.1097/00001756-199110000-00006

Einstein, E. B., Patterson, C. A., Hon, B. J., Regan, K. A., Reddi, J., Melnikoff, D. E., et al. (2010). Somatostatin signaling in neuronal cilia is critical for object recognition memory. J. Neurosci. 30, 4306-4314. doi: 10.1523/JNEUROSCI. 5295-09.2010

Engler-Chiurazzi, E. B., Singh, M., and Simpkins, J. W. (2016). From the 90's to now: a brief historical perspective on more than two decades of estrogen neuroprotection. Brain Res. 1633, 96-100. doi: 10.1016/j.brainres.2015.12.044

Ferreira-Vieira, T. H., Guimaraes, I. M., Silva, F. R., and Ribeiro, F. M. (2016). Alzheimer's disease: targeting the cholinergic system. Curr. Neuropharmacol. 14, 101-115. doi: 10.2174/1570159x13666150716165726

Gallina, P., Paganini, M., Lombardini, L., Saccardi, R., Marini, M., De Cristofaro, M. T., et al. (2008). Development of human striatal anlagen after transplantation in a patient with Huntington's disease. Exp. Neurol. 213, 241-244. doi: 10.1016/j.expneurol.2008.06.003

Geula, C., Schatz, C. R., and Mesulam, M. M. (1993). Differential localization of NADPH-diaphorase and calbindin-D28k within the cholinergic neurons of the basal forebrain, striatum and brainstem in the rat, monkey, baboon and human. Neuroscience 54, 461-476. doi: 10.1016/0306-4522(93)90266-i

Gibbs, R. B., Nelson, D., and Hammond, R. (2014). Role of GPR30 in mediating estradiol effects on acetylcholine release in the hippocampus. Horm. Behav. 66, 339-345. doi: 10.1016/j.yhbeh.2014.06.002

Giovannini, M. G., Lana, D., and Pepeu, G. (2015). The integrated role of ACh, ERK and mTOR in the mechanisms of hippocampal inhibitory avoidance memory. Neurobiol. Learn. Mem. 119, 18-33. doi: 10.1016/j.nlm.2014.12.014

González-Martínez, D., Kim, S. H., Hu, Y., Guimond, S., Schofield, J., Winyard, P., et al. (2004). Anosmin-1 modulates fibroblast growth factor receptor 1 signaling in human gonadotropin-releasing hormone olfactory neuroblasts through a heparan sulfate-dependent mechanism. J. Neurosci. 24, 10384-10392. doi: 10.1523/JNEUROSCI.3400-04.2004

Gorry, J. D. (1963). Studies on the comparative anatomy of the Ganglion Basale of Meynert. Acta Anat. 55, 51-104. doi: 10.1159/000142464

Gotti, C., Moretti, M., Gaimarri, A., Zanardi, A., Clementi, F., and Zoli, M. (2007). Heterogeneity and complexity of native brain nicotinic receptors. Biochem. Pharmacol. 74, 1102-1111. doi: 10.1016/j.bcp.2007.05.023

Grealish, S., Diguet, E., Kirkeby, A., Mattsson, B., Heuer, A., Bramoulle, Y., et al. (2014). Human ESC-derived dopamine neurons show similar preclinical efficacy and potency to fetal neurons when grafted in a rat model of Parkinson's disease. Cell Stem Cell 15, 653-665. doi: 10.1016/j.stem.2014.09.017

Grossi, C., Francese, S., Casini, A., Rosi, M. C., Luccarini, I., Fiorentini, A., et al. (2009). Clioquinol decreases amyloid- $\beta$ burden and reduces working memory impairment in a transgenic mouse model of Alzheimer's disease. J. Alzheimers Dis. 17, 423-440. doi: 10.3233/JAD-2009-1063

Grossi, C., Rigacci, S., Ambrosini, S., Ed Dami, T., Luccarini, I., Traini, C., et al. (2013). The polyphenol oleuropein aglycone protects TgCRND8 mice against Aßplaque pathology. PLoS One 8:e71702. doi: 10.1371/journal.pone.0071702
Hammond, R., and Gibbs, R. B. (2011). GPR30 is positioned to mediate estrogen effects on basal forebrain cholinergic neurons and cognitive performance. Brain Res. 1379, 53-60. doi: 10.1016/j.brainres.2010.11.098

Han, Y. G., Spassky, N., Romaguera-Ros, M., Garcia-Verdugo, J. M., Aguilar, A., Schneider-Maunoury, S., et al. (2008). Hedgehog signaling and primary cilia are required for the formation of adult neural stem cells. Nat. Neurosci. 11, 277-284. doi: 10.1038/nn2059

Händel, M., Schulz, S., Stanarius, A., Schreff, M., Erdtmann-Vourliotis, M., Schmidt, H., et al. (1999). Selective targeting of somatostatin receptor 3 to neuronal cilia. Neuroscience 89, 909-926. doi: 10.1016/s0306-4522(98)00354-6

Hellström-Lindahl, E., Gorbounova, O., Seiger, A., Mousavi, M., and Nordberg, A. (1998). Regional distribution of nicotinic receptors during prenatal development of human brain and spinal cord. Dev. Brain Res. 108, 147-160. doi: 10.1016/s0165-3806(98)00046-7

Inanobe, A., and Kurachi, Y. (2014). Membrane channels as integrators of G-protein-mediated signaling. Biochim. Biophys. Acta 1838, 521-531. doi: 10.1016/j.bbamem.2013.08.018

Janis, L. S., Glasier, M. M., Fulop, Z., and Stein, D. G. (1998). Intraseptal injections of $192 \mathrm{IgG}$ saporin produce deficits for strategy selection in spatial-memory tasks. Behav. Brain Res. 90, 23-34. doi: 10.1016/s0166-4328(97)00078-8

Jorm, A. F., and Jolley, D. (1998). The incidence of dementia: a meta-analysis. Neurology 51, 728-733. doi: 10.1212/WNL.51.3.728

Kilimann, I., Grothe, M., Heinsen, H., Alho, E. J., Grinberg, L., Amaro, E. Jr., et al. (2014). Subregional basal forebrain atrophy in Alzheimer's disease: a multicenter study. J. Alzheimers Dis. 40, 687-700. doi: 10.3233/JAD-132345

Koliatsos, V. E., Clatterbuck, R. E., Nauta, H. J., Knüsel, B., Burton, L. E., Hefti, F. F., et al. (1991). Human nerve growth factor prevents degeneration of basal forebrain cholinergic neurons in primates. Ann. Neurol. 30, 831-840. doi: 10.1002/ana.410300613

Kostović, I. (1986). Prenatal development of nucleus basalis complex and related fiber systems in man: a histochemical study. Neuroscience 17, 1047-1077. doi: 10.1016/0306-4522(86)90077-1

Kračun, I., and Rösner, H. (1986). Early Cytoarchitectonic development of the anlage of the basal nucleus of Meynert in the human fetus. Int. J. Dev. Neurosci. 4, 143-149. doi: 10.1016/0736-5748(86)90039-0

Lee, S. T., Chu, K., Park, J. E., Lee, K., Kang, L., Kim, S. U., et al. (2005). Intravenous administration of human neural stem cells induces functional recovery in Huntington's disease rat model. Neurosci. Res. 52, 243-249. doi: 10.1016/j. neures.2005.03.016

Lee, J. E., Jeong, D. U., Lee, J., Chang, W. S., and Chang, J. W. (2016). The effect of nucleus basalis magnocellularis deep brain stimulation on memory function in a rat model of dementia. BMC Neurol. 16:6. doi: 10.1186/s12883-016-0529-z

Lepski, G., Maciaczyk, J., Jannes, C. E., Maciaczyk, D., Bischofberger, J., and Nikkhah, G. (2011). Delayed functional maturation of human neuronal progenitor cells in vitro. Mol. Cell. Neurosci. 47, 36-44. doi: 10.1016/j.mcn.2011. 02.011

Liu, Y., Weick, J. P., Liu, H., Krencik, R., Zhang, X., Ma, L., et al. (2013). Medial ganglionic eminence-like cells derived from human embryonic stem cells correct learning and memory deficits. Nat. Biotechnol. 31, 440-447. doi: $10.1038 /$ nbt. 2565

Martínez-Morales, P. L., Revilla, A., Ocaña, I., González, C., Sainz, P., McGuire, D., et al. (2013). Progress in stem cell therapy for major human neurological disorders. Stem Cell Rev. 9, 685-699. doi: 10.1007/s12015-013-9443-6

Matucci, R., Nesi, M., Martino, M. V., Bellucci, C., Manetti, D., Ciuti, E., et al. (2016). Carbachol dimers as homobivalent modulators of muscarinic receptors. Biochem. Pharmacol. 108, 90-101. doi: 10.1016/j.bcp.2016.03.012

Mesulam, M. M. (2013). Cholinergic circuitry of the human nucleus basalis and its fate in Alzheimer's disease. J. Comp. Neurol. 521, 4124-4144. doi: 10.1002/cne. 23415

Morelli, A., Comeglio, P., Filippi, S., Sarchielli, E., Vignozzi, L., Maneschi, E., et al. (2013). Mechanism of action of phosphodiesterase type 5 inhibition in metabolic syndrome-associated prostate alterations: an experimental study in the rabbit. Prostate 73, 428-441. doi: 10.1002/pros.22584

Morelli, A., Marini, M., Mancina, R., Luconi, M., Vignozzi, L., Fibbi, B., et al. (2008). Sex steroids and leptin regulate the "first Kiss" (KiSS 1/G-proteincoupled receptor 54 system) in human gonadotropin-releasing-hormonesecreting neuroblasts. J. Sex. Med. 5, 1097-1113. doi: 10.1111/j.1743-6109.2008. 00782.x 
Mufson, E. J., Ginsberg, S. D., Ikonomovic, M. D., and DeKosky, S. T. (2003). Human cholinergic basal forebrain: chemoanatomy and neurologic dysfunction. J. Chem. Neuroanat. 26, 233-242. doi: 10.1016/s08910618(03)00068-1

Paganini, M., Biggeri, A., Romoli, A. M., Mechi, C., Ghelli, E., Berti, V., et al. (2014). Fetal striatal grafting slows motor and cognitive decline of Huntington's disease. J. Neurol. Neurosurg. Psychiatry 85, 974-981. doi: 10.1136/jnnp-2013306533

Paxinos, G., and Watson, C. (2006). The Rat Brain in Stereotaxic Coordinates. London: Academic Press.

Perini, G., Della-Bianca, V., Politi, V., Della Valle, G., Dal-Pra, I., Rossi, F., et al. (2002). Role of p75 neurotrophin receptor in the neurotoxicity by $\beta$-amyloid peptides and synergistic effect of inflammatory cytokines. J. Exp. Med. 195, 907-918. doi: 10.1084/jem.20011797

Pombero, A., Bueno, C., Saglietti, L., Rodenas, M., Guimera, J., Bulfone, A., et al. (2011). Pallial origin of basal forebrain cholinergic neurons in the nucleus basalis of Meynert and horizontal limb of the diagonal band nucleus. Development 138, 4315-4326. doi: 10.1242/dev.069534

Ramirez-Latorre, J., Yu, C. R., Qu, X., Perin, F., Karlin, A., and Role, L. (1996). Functional contributions of $\alpha 5$ subunit to neuronal acetylcholine receptor channels. Nature 380, 347-351. doi: 10.1038/380347a0

Revankar, C. M., Cimino, D. F., Sklar, L. A., Arterburn, J. B., and Prossnitz, E. R. (2005). A transmembrane intracellular estrogen receptor mediates rapid cell signaling. Science 307, 1625-1630. doi: 10.1126/science. 1106943

Rodríguez, J. J., Jones, V. C., Tabuchi, M., Allan, S. M., Knight, E. M., LaFerla, F. M., et al. (2008). Impaired adult neurogenesis in the dentate gyrus of a triple transgenic mouse model of Alzheimer's disease. PLoS One 3:e2935. doi: 10.1371/journal.pone.0002935

Sah, D. W. (1995). Human fetal central neurons in culture: voltage- and ligandgated currents. J. Neurophysiol. 74, 1889-1899.

Sarchielli, E., Comeglio, P., Squecco, R., Ballerini, L., Mello, T., Guarnieri, G., et al. (2017). Tumor necrosis factor- $\alpha$ impairs kisspeptin signaling in human gonadotropin-releasing hormone primary neurons. J. Clin. Endocrinol. Metab. 102, 46-56. doi: 10.1210/jc.2016-2115

Sarchielli, E., Marini, M., Ambrosini, S., Peri, A., Mazzanti, B., Pinzani, P., et al. (2014). Multifaceted roles of BDNF and FGF2 in human striatal primordium development. An in vitro study. Exp. Neurol. 257, 130-147. doi: 10.1016/j. expneurol.2014.04.021

Shen, C. C., Lin, C. H., Yang, Y. C., Chiao, M. T., Cheng, W. Y., and Ko, J. L. (2010). Intravenous implanted neural stem cells migrate to injury site, reduce infarct volume, and improve behavior after cerebral ischemia. Curr. Neurovasc. Res. 7, 167-179. doi: 10.2174/156720210792231822

Song, M., Mohamad, O., Chen, D., and Yu, S. P. (2013). Coordinated development of voltage-gated $\mathrm{Na}^{+}$and $\mathrm{K}^{+}$currents regulates functional maturation of forebrain neurons derived from human induced pluripotent stem cells. Stem Cells Dev. 22, 1551-1563. doi: 10.1089/scd.2012.0556

Torres, E. M., Perry, T. A., Blockland, A., Wilkinson, L. S., Wiley, R. G., Lappi, D. A., et al. (1994). Behavioural, histochemical and biochemical consequences of selective immunolesions in discrete regions of the basal forebrain cholinergic system. Neuroscience 63, 95-122. doi: 10.1016/03064522(94)90010-8

Tuszynski, M. H., Thal, L., Pay, M., Salmon, D. P., U, H. S., Bakay, R., et al. (2005). A phase 1 clinical trial of nerve growth factor gene therapy for Alzheimer disease. Nat. Med. 11, 551-555. doi: 10.1038/nm1239

Wonnacott, S., Barik, J., Dickinson, J., and Jones, I. W. (2006). Nicotinic receptors modulate transmitter cross talk in the CNS: nicotinic modulation of transmitters. J. Mol. Neurosci. 30, 137-140. doi: 10.1385/jmn: 30:1:137

Xu, Q., Tam, M., and Anderson, S. A. (2008). Fate mapping Nkx2.1-lineage cells in the mouse telencephalon. J. Comp. Neurol. 506, 16-29. doi: 10.1002/cne. 21529

Xu, W., Tan, L., Wang, H. F., Jiang, T., Tan, M. S., Tan, L., et al. (2015). Metaanalysis of modifiable risk factors for Alzheimer's disease. J. Neurol. Neurosurg. Psychiatry 86, 1299-1306. doi: 10.1136/jnnp-2015-310548

Yue, W., Li, Y., Zhang, T., Jiang, M., Qian, Y., Zhang, M., et al. (2015). ESCderived basal forebrain cholinergic neurons ameliorate the cognitive symptoms associated with Alzheimer's disease in mouse models. Stem Cell Reports 5, 776-790. doi: 10.1016/j.stemcr.2015.09.010

Conflict of Interest Statement: The authors declare that the research was conducted in the absence of any commercial or financial relationships that could be construed as a potential conflict of interest.

Copyright (C) 2017 Morelli, Sarchielli, Guarnieri, Coppi, Pantano, Comeglio, Nardiello, Pugliese, Ballerini, Matucci, Ambrosini, Castronovo, Valente, Mazzanti, Bucciantini, Maggi, Casamenti, Gallina and Vannelli. This is an open-access article distributed under the terms of the Creative Commons Attribution License (CC BY). The use, distribution or reproduction in other forums is permitted, provided the original author(s) or licensor are credited and that the original publication in this journal is cited, in accordance with accepted academic practice. No use, distribution or reproduction is permitted which does not comply with these terms. 